\title{
A Preliminary SKOS Implementation of the Art and Architecture Thesaurus: Machine-Actionable Controlled Vocabulary for the Semantic Web
}

Eric A. Robinson

University of St. Augustine for Health Sciences, erobinson@usa.edu

Author(s) ORCID Identifier:

(iD) https://orcid.org/0000-0001-9554-8754

Follow this and additional works at: https://soar.usa.edu/other

\section{Recommended Citation}

Robinson, Eric A., "A Preliminary SKOS Implementation of the Art and Architecture Thesaurus: MachineActionable Controlled Vocabulary for the Semantic Web" (2011). Other Topics. 13.

https://soar.usa.edu/other/13 


\section{A Preliminary SKOS Implementation of the Art and Architecture Thesaurus: Machine-Actionable Controlled Vocabulary for the Semantic Web}

Eric A. Robinson, MLIS ${ }^{1}$

iD https://orcid.org/0000-0001-9554-8754

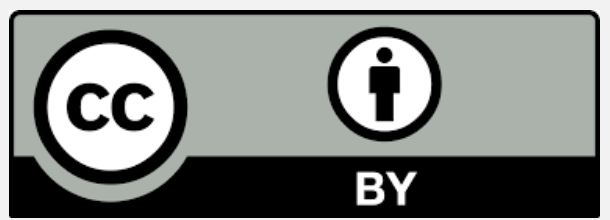

"A Preliminary SKOS Implementation of the Art and Architecture Thesaurus: Machine-Actionable Controlled Vocabulary for the Semantic Web" by Eric A. Robinson is licensed under a Creative Commons Attribution 4.0 International License.

This license gives advance permission to copy and redistribute this material in any medium or format; to remix, transform, and build upon the material for any purpose, even commercially; provided appropriate credit is given to the author, including a link to the CC-BY license, and the original work, and indicating the changes, if any, that were made. You may do so in any reasonable manner, but not in any way that suggests the author endorses you or your use.

Abstract: This paper presents an experimental implementation of the Art and Architecture Thesaurus, a knowledge organizational system for the visual arts and architecture, within the SKOS (Simple Knowledge Organization System) framework. Such treatment allows for machine-actionability on thesaurus records for automated expansion of search queries and also provides a framework for interoperability across metadata schemas in a linked data environment. SKOS enables more complex semantic processing by utilizing a simple framework for Semantic Web technology within thesauri. The analysis establishes an application profile for AAT, which accommodates the faceted and polyhierarchical structure of the thesaurus, as well as the detailed source referencing contained within AAT's documentary notes.

\section{Introduction}

In order to utilize metadata schemas in the thoroughly linked information environment of the 21st century, developers must pursue standards that help to bring together the various schemas in existence and to facilitate coding of metadata that can accommodate machineactionability on linked data in a Semantic Web framework. The diverse communities that have driven context specific schemas often look to standardized schemas like Dublin Core, MODS or VRA,

1 University of St. Augustine for Health Sciences

Correspondence email: erobinson@usa.edu 
and employ standardized vocabularies like the Library of Congress Subject Headings for classification purposes. By incorporating semantic web technologies into these standards, information organizations can better facilitate cross-repository searching, and complex query expansions.

The Simple Knowledge Organization System (SKOS) provides a format for encoding many of the existing thesauri, ontologies, and other knowledge organization systems in a highly machinereadable format. This system can thus act as a modular expansion of many of the metadata frameworks already in existence, conferring upon them greater interoperability, and expanding efforts to link data between divergent information repositories. As SKOS is based upon the Semantic Web enabled Resource Description Framework (RDF), it also allows for inferential processing of classification metadata, which an enormous step for more intelligent searching, retrieval, and query expansion.

The Art and Architecture Thesaurus (AAT), a specialized vocabulary for the visual arts and architectural fields is a well-established standard for vocabulary control. It is at first glance an ideal candidate for representation in SKOS. It incorporates many international thesaurus standards such as syndetic broader and narrow term references, hierarchical relationships, preferred terminology for vocabulary control, and associative relationships for query expansion. However, a preliminary experimental implementation of AAT concepts and hierarchies within the SKOS framework indentified a number of challenges. After a review of relevant literature describing the foundational concepts of SKOS, this paper will detail the challenges and potential proposals for a fuller semantic representation of AAT within SKOS.

\section{Review of Literature}

Since early discussion of the potential for the Semantic Web to improve searchability and machine-processing of web resources (Berners-Lee et al., 2001), efforts have been made to develop metadata schemas that are more in line with the standards of semantic processing. The most critical element for the capture of meaning in this context is the description of relationships among resources. By describing such relationships with a clear, expressive syntax, applications can be applied to metadata descriptions that incorporate logical inference to enrich the process. SKOS enables the use of a Semantic Web framework to describe knowledge organization systems (KOS) for the easy publication, sharing, and re-use of these systems on the Web. SKOS is built upon foundational data formats and schemas, including XML, RDF and RDF Schema (RDF-S) and confers Semantic Web capabilities on the data, allowing for logical inference across defined concepts. These 
elements have been more thoroughly reviewed elsewhere (Robinson, ms.), and discussion proceeds without further explanation of Semantic Web foundations.

SKOS builds on the simplified class and property descriptions of RDF-S, to describe standards identified for thesauri, classification schemata, and other knowledge organization systems (A. Miles, Matthews, Wilson, \& D. Brickley, 2005).

SKOS began as a RDF Schema framework by the to advance the Semantic Web effort in Europe. This early version, known as the DESIRE project (Lacasta, Nogueras-Iso, Lopez-Pellicer, Muro-Medrano, \& Zarazaga-Soria, 2007; A. Miles et al., 2005), was undertaken by the Semantic Web Advanced Development group for Europe (SWAD-E) and was intended to represent a generic thesaurus representation for the Semantic Web. DESIRE was elaborated and improved upon as LIMBER, a domain specific knowledge organization system for the social sciences. LIMBER incorporated standard guidelines for the creation of thesauri such as those conceived by ANSI/NISO and related organizations (International Organization for Standardization, 1985, 1986) and incorporated translational elements for use in international metadata effort to allow queries in a user's own language (A. Miles et al., 2005). Since 2004, development has continued under the auspices of the W3C Semantic Web Best Practices and Deployment Working Group (SWBPDWG) and enlists the vast efforts of the open development community (Bueno de la Fuente, 2008).

While other languages, such as OWL and DAML have been developed with very rich ontological frameworks (McGuinness \& van Harmelen, 2004) SKOS is intended to capture the basics of KOS ontologies in a simpler and more widely employable framework. As Mikhalenko has described it, SKOS is intended to fill the "need for a language to express vocabularies of concepts for use in semantically rich metadata, which is powerful enough to support semantically enhanced search, but simple enough to be undemanding in terms of the cost and expertise required to use it." (Mikhalenko, 2005, par. 5).

It is composed of three separate specifications, the SKOS Core vocabulary, SKOS Mapping, and SKOS extension. SKOS Core provides the basic vocabularies necessary to describe the hierarchical structures, class dependencies, and properties important for the representation of a KOS (A. Miles et al., 2005). The core specification also provides documentation vocabulary, such as scope notes to elaborate upon the precise intention for use of concepts in the KOS, and historical notes for tracing changes to a specific implementation. The SKOS Mapping specification is a reference guide for supporting alignment and linking between different KOS concept schemes (Alistair Miles \& Bechhofer, 2009; Alistair Miles \& Dan Brickley, 2004). Finally, SKOS Extension represents properties and relations peculiar to only some KOS (Lacasta et al., 2007). These are 
often unique adaptations to a particular KOS need within a specific domain or schema implementation.

The concepts comprising any thesaurus implementation in SKOS are represented using a few simple properties to describe the nodes of the thesaurus ontology. The ontological relations of the thesaurus, being hierarchical in nature can be viewed as a branching tree structure with each concept class being treated as a node in the structure. The RDF-S subclassOf property is used to describe the relations of narrower terms in the hierarchy, and the elements of the KOS themselves are treated as a subclass of the concept scheme, using the inscheme property. Each term is treated as a concept and its properties are described using SKOS terminology in an RDF style document. These properties include syndetic class relations or hierarchical, semantic relationships, label preferences, and documentary notations.

The hierarchical relationships of the thesaurus such as those represented in a thesaurus as broader and narrower terms are captured with the properties skos:broader and skos: narrower. Non-hierarchical, or simple semantic associative relationships, such as "see also", are expressed via the skos: related property (Isaac \& Summers, 2009; Alistair Miles \& Bechhofer, 2009). Statements are made in the usual RDF fashion by declaring properties to be about a concept, represented as a particular URI, and then enumeration the properties of that concept in SKOS. This is useful for the computational recognition of potentially related search terms, and can be employed either to present potential terms to a user, or to automatically broaden a search if necessary.

Expressed in RDF-style syntax then, the basic semantic relationships can be captured as triples in RDF syntax as in figure 1, shown without namespace declarations. The $\mathrm{xx} x \#$ descriptions in this example and in the examples in this paper would typically be replaced by form of unique identifier, such as an authority system control number.

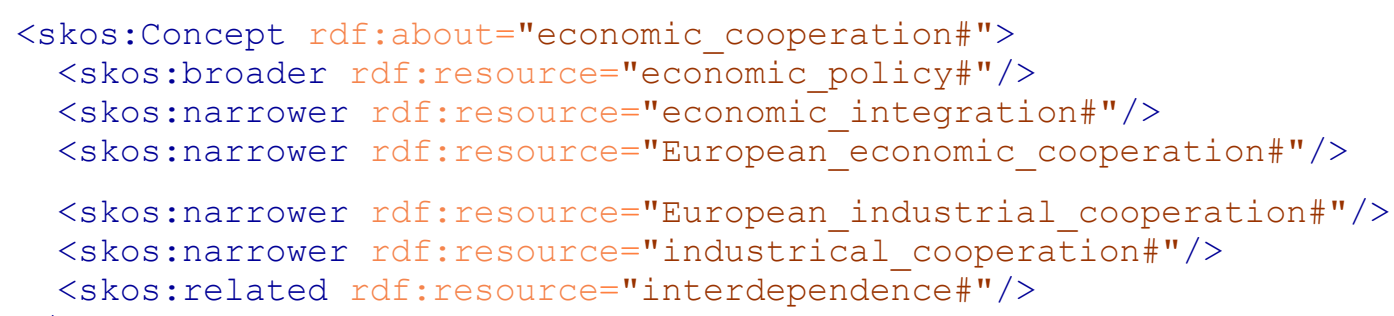

Fig. 1 Hierarchical relationships in SKOS 
Term labels in SKOS allow the expression of preferred terminology and the capture of alternate terminologies. For example, the above "Economic Cooperation" entry might also be sought as "Economic Co-operation" This could be expressed using the a lt Label and pref Label properties (Isaac \& Summers, 2009). SKOS also contains a property called hiddenLabel for capturing common misspellings. This capability allows recognition of the access points that might be attempted, but that the programmer does not want to appear to the public (Alistair Miles \& Bechhofer, 2009). This could be useful for linking to commonly misspelled names, beyond the scope of alternate, but legitimate names, often used in name authority files. Thus a potential misspelling of Mark Twain's autonym, and the pseudonym itself might be captured as in figure 2 .

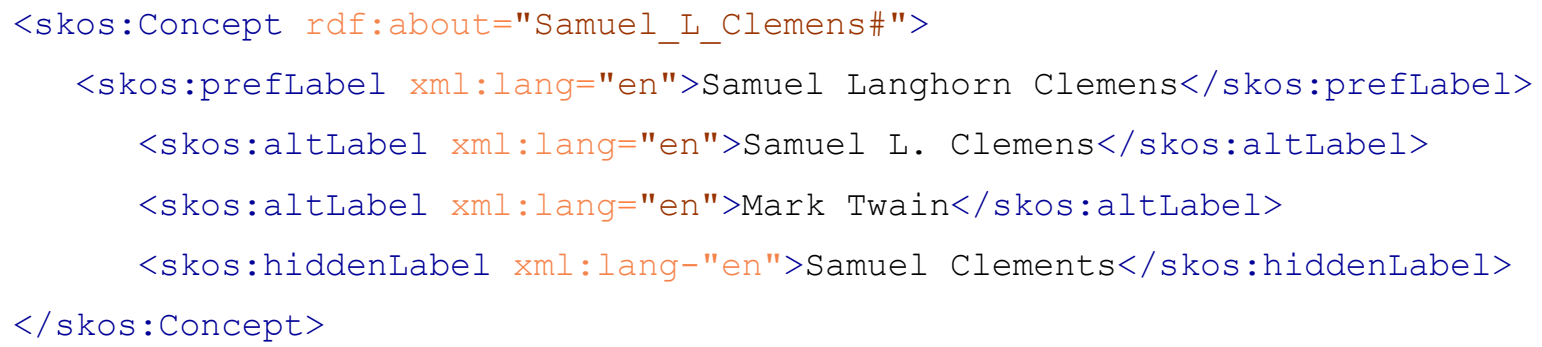

Fig. 2 Preferred and alternate terms in SKOS

AltLabel can also be used to incorporate interlingual elements into SKOS by related translated terms to a single declared URI concept. These interlingual elements would then be identified with an appropriate $\mathrm{xml}:$ lang attribute. This allows for even greater integration and reusability, since metadata descriptors throughout the international community can utilize and build upon single SKOS concepts, fostering interoperability on a worldwide scale.

Finally, the SKOS Core specifications also allow for documentary descriptions. These descriptions can define textual content to describe intended use and scope and also permit a modest level of administrative detail regarding the developmental history of the schema. The NISO guidelines for thesaurus creation recognizes the need to clearly distinguish uses which may be ambiguous, or to identify that particular range of scope intended to be covered by term (International Organization for Standardization, 1985, 1986). SKOS utilizes the skos: definition property to define the scope of a concept and to clarify coverage of a particular concept term.

Applications of SKOS in the Web Metadata Environment 
By encoding these relationships, both hierarchical and terminological, in RDF, the vocabulary control process and hierarchical relationships of ontologies can be readily leveraged into the online searching environment to increase searchability and interoperability and to promote precision and recall (Antoniou \& Harmelen, 2008). Applications and processors can be written that refer to the published namespaces and schemata contained therein, combining defined KOS into other metadata schemata like Dublin Core (DC) and the Metadata Object Description Standard (MODS). The easily linkable nature of SKOS schema also allows the large computational resources of the web to be brought to bear upon the navigational structure of the web, enriching search processes by increasing interoperability and allowing cross-searching of a vast number of resource repositories.

One of the most significant applications of SKOS and RDF in the web environment is the so called "Linked Data" movement. A sort of rebranding of the Semantic Web, linked data builds upon the RDF-style expressions described with a focus on the connection and exposure of data within documents, rather than the simple linking between documents themselves using hyperlinks (Bizer, Heath, Ayers, \& Raimond, n.d.). Such a focus is contrasted with the hyperlinks of the traditional web, by referring to this data as "hyperdata" (Bizer, R. Cyganiak, \& Heath, 2008). By structuring and labeling the data using Semantic Web technologies, it is extracted and made more accessible, allowing connections between a wide variety of froms. For example as Bizer et al. describe, "Using these links one can navigate from a computer scientist in dbpedia to her publications in the DBLP database, from a dbpedia book to reviews and sales offers for this book provided by the RDF Book Mashup, or from a band in dbpedia to a list of their songs provided by Musicbrainz or dbtune" (n.d., para. 4)

The Library of Congress (LC) has recently made tremendous efforts in this regard, and in 2009, it bagan to make its ubiquitously employed authority records available as linked data (“Authorities \& Vocabularies (Library of Congress)"; Bradley, 2009; Karen Coyle, 2009; Harper \& Tillett, 2007). Its primary goal is to enable data access via dereferencable URIs in the form of SKOS encodings. This allows the LC's controlled vocabularies and the data values that they comprise. Thus, creators of content or programmers who build metadata processors can incorporate LC metadata as linked data. The vocabularies themselves are also readily made available in a webpublishable format for easy vocabulary minting, updating, and downloading ("Authorities \& Vocabularies (Library of Congress)”). 
The new "webified" LC Authorities and Vocabularies, by being published in SKOS allow for more rapid updating of systems that employ this data, and also provide cost-free access far superior to the days of the "Big Red Books", the paper issue of the Library of Congress Subject Headings.

Other efforts for utilizing SKOS on the Semantic Web front include projects to incorporate controlled vocabularies into the organization and classification of user generated content and the incorporation of federated search standards. One of the standard methodologies for mapping across metadata schemas is the employment of an intermediary linking standard, a switching language, to which equivalent terms are converted (Zeng \& Chan, 2004). These anchor terms serve as a master language then, for the conversion of multiple KOS. The ability of SKOS to represent a wide range of alternate terminology and its potential to capture a range of schema through RDF and XML namespace representation make it an ideal "interlingua” for KOS interoperability.

Tudhope and Binding have examined the efforts of the STAR Project, a massive integration of English Heritage thesauri, for its employment of SKOS as a standard conversion format (Tudhope \& Binding, 2008). Efforts have been also made to establish effective procedures for the automated conversion of many different thesauri into SKOS under the auspices of the W3C's Semantic Web Best Practices Working Group (Van Assem, Malaisé, A. Miles, \& Schreiber, 2006)

The STAR (Semantic Technologies for Archaeological Resources) Project utilized SKOS in this way to create a search interface for federated search of seven different thesauri encompassing archaeological, materials, and buildings and monuments indices employed in the mapping of the broad domain of English Heritage. It allowed for the creation of a multifunctional interface incorporating standard search procedures, search term suggestions, and query expansion based on the related hierarchies in the thesaurus array. Explorable concept schemes were also generated for user navigation and linking to relevant documents (Binding, May, \& Tudhope, 2008; Tudhope, Binding, May, \& Heritage, 2008). The success of the STAR project showcases the ability for SKOS to serve as a switching language for interoperability on a grander scale. The creation of such interfaces is an enormous, but necessary step for metadata technology if libraries are to leverage the multiple silos of data that exist on the web and to bring them under the umbrella of federated searching projects.

The range of isolated information repositories and the variety of control schemas employed is only one of the problems posed by metadata searching in the web environment. Since about 2004, new social networking technologies have begun to employ user-generated tagging to provide quick labeling of content under interactive content models of Web 2.0. Websites such as Flickr or del.icio.us began the trend, but now even commonly accessed news resources regularly allow users 
to label content with whatever terminology they might find useful for their own reference and to maintain lists of tags affiliated with their user accounts. While this creates a vast repository of incredibly inexpensive metadata for the rapidly generated content of the Web, the lack standards and control in these systems has been criticized. It is widely accepted that these folksonomies display an inability to deal with concepts of synonomy, variant usage, and spelling, and, at least in their native form, are difficult to utilize for accurate information retrieval (Limpens, Gandon, \& Buffa, 2009). As MacGregor and McCulloch state, "to ensure effective indexing and to maintain the overall efficacy of the retrieval system, it is necessary to apply some degree of control to the indexing process" (2006, p. 292).

As described above, the labels employed for SKOS concepts can be broadened to incorporate the widely divergent vocabularies employed under social tagging, thus linking them to more usable controlled KOS (Isaac \& Summers, 2009). By employing records which merge usergenerated tag lists with representative control data, online searches can be expanded in a controlled way to retrieve relevant information with semantic enhancement of tag-style metadata. Tagging software might be written to incorporate simple selection processes which facilitate linking to controlled concept URIs. SCOT (Social Semantic Cloud of Tags), an RDF-based ontology has been developed as an extension of SKOS intended to capture the structure and semantics of tagging systems. The declared intention of SCOT is to create repurposable semantic data for use in federating existing folksonomies (Kim, Passant, Breslin, Scerri, \& Decker, 2008).

Similar efforts have been made by Simon Jupp and his colleagues at the Sealife project to incorporate a large number of biomedical ontologies into a single accessible SKOS framework. Their Conceptual Open Hypermedia Service (COHSE) eschews the richer ontology languages like OWL for the simpler representation of SKOS, since it allows them to incorporate semantically weaker structures like thesauri into the COHSE system (Jupp, Stevens, Bechhofer, Yesilada, \& Kostkova, 2008). Their project uses linked data coded in SKOS to identify background knowledge represented in a repository of web linked documentation. By identifying appropriate content via existing KOS, COHSE is able to support semantic web navigation through the specialized Sealife semantic web browser. Sealife utilizes the ontologies to mechanically markup documents with semantic encoding dynamically at the time of browsing. Thus, without prior semantic preparation, which can be time consuming and expensive, semantic technologies can be leveraged to identify key content in a document and offer links to appropriate services from the browse site.

As exemplified in these projects, SKOS is a technological tool that can offer rich benefits to information organization and search. By defining concept schemes that can be related across 
organizations, SKOS bridges the gaps of information silos, as in the STAR project. It also allows for a rich foundation for query expansion based up intelligent semantic inferential processes. Implementation of SKOS enabled systems in standard knowledge organization frameworks, like thesauri, ontologies, and classification systems, then, would offer enormous benefits to information organization and retrieval.

\section{Art and Architecture Thesaurus}

The Art and Architecture Thesaurus (AAT) is developed and maintained by the Getty Center and has been in use since its initial development in the 1970s. Originally intended for the use of art libraries and journals, the AAT provides a standard for controlled vocabulary metadata in the fields of visual arts and architecture ("Art and Architecture Thesaurus Online," 2010). The thesaurus provides a structured hierarchy of preferred terminology and captures semantic relationships across terms. Built upon international standards for thesaurus development the AAT includes listings for broader and narrower terms, associative relationships, scope descriptions and notes outlining the sources for the establishment of preferred terminology. While many of thesaurus properties are standard and easily represented within the SKOS framework, others pose challenges and offer opportunities for development and expansion of SKOS via additional extensions. After a brief discussion of the structure and scope of the AAT, results of a preliminary study for the development of a SKOS application profile for AAT will be presented.

The AAT provides standard terminological relationships captured in any thesaurus utilizing international development standards. Broader and narrower relationships are clearly described, establishing hierarchies of progressively specific concept terms. Related terms, which refer to semantically associated concepts outside the semantic hierarchy, are included for each concept, allowing for associative query expansion. These relations are part of the standard SKOS framework, and are easily represented without any special accommodations.

The terminology utilized within the AAT derives from the preferred usage within the art and architecture community. Strong efforts are made to document sources both for the range of usage and for the establishment of a pattern of preferred usage. Source notes are used to provide references to standard works, journals, and indexes relevant to a given term. While SKOS does provide a framework for documentary notes, the specificity of the source notes in AAT require 
further development of that framework to distinguish sources of preferred usage and to make those references more semantically actionable.

In order to deal with the problem of homonyms, AAT utilizes qualifiers with some concepts to distinguish between essential variants. For example, the 'museums' concept can be distinguished by the abstract institution or by the building itself. Each of the two concepts will be represented as a distinct entry in AAT with a qualifier clarifying the context of the entry. As AAT explicitly requires that this qualifier not be listed as a part of the preferred term for the element, the qualifying context must be associated with the concept elsewhere in the record.

AAT also displays very intricate structural relationships between terms and concepts in the thesaurus. Most thesauri utilize some level of hierarchy in their structure that provides for the grouping of homogeneous concepts into broader concept categories, similar to the genus/species relationships in biological taxonomy. Thus, a broad class of 'animals' may include the concepts of 'mammals', 'reptiles', and 'fishes', while 'mammals' circumscribes the multiple classes of mammals, cats, dogs, cows, humans, etc.

AAT utilizes these standard hierarchical concepts, but further broadens them to include polyhierarchical relationships and faceted classification. Polyhierarchy refers to an arrangement where any thesaurus concept may be viewed as a narrower division of more than one broader parent concept. Thus in AAT, the concept term 'graffiti' may be organized under both the broader concepts of 'information forms' and 'visual works'. It could not then be viewed strictly as a descendant of either of these. Rather there is a need to represent its membership in both, while recognizing a preferred structure.

Within a strict formal semantic ontology, this ambiguity could prove problematic. As logical functions operate on the principles of transitivity within these hierarchies, the multiple parent nodes present in polyhierarchical relationships of AAT could lead to semantic contradiction, or at least semantic ambiguities. Thus a method of representing preferred hierarchical relationships would be beneficial for computations on narrower and broader classes.

AAT also utilizes a complex arrangement of faceted classification within its hierarchy. This can be viewed as a more specific instance of the polyhierarchy discussed above. Like hierarchical concepts, facets provide a way of classifying a group of homogenous concepts that share some property. Facets often bridge simple hierarchical relationships and bundle a relevant selection of properties under an overarching heading. AAT utilizes a rich set of facets in a number of categories with hierarchical sets nested within:

- Associated Concepts-e.g., 
- Physical Attributes-including Attributes and Properties, Conditions and Effects, Design Elements, and Colors

- Styles and Periods

- Agents-including People, Organizations, and Living Organisms

- Activities--

- Materials

- Objects

The Objects facet is by far the broadest and most inclusive. Thus it also includes nested subfacets such as 'built environment' and 'furnishings and equipment'. Many of the concepts for architectural buildings and components fall within this facet and SKOS representation of these elements becomes critical in that context.

\section{Implementation}

In order to explore the possibilities and challenges of implementing the Art and Architecture Thesaurus in SKOS, twenty-five AAT concept terms from a range of hierarchical levels and subject topics pertinent to the field of architecture were translated into full SKOS records.

Though AAT does cover many more topics in the visual arts, the narrower scope of architecture allowed greater attention to be given to a narrow range of facets and concepts, allowing more effective preliminary formulations. It is expected that the models derived from these narrow topics would be easily expanded to incorporate the various concepts, labels, and facets required for full representation of all AAT concepts.

SKOS records for each term were created using a standard RDF framework represented in XML. The Oxygen XML Editing Environment was utilized for writing the XML code. This environment aids in the identification of invalid XML code, and was of great assistance in constraining proposed solutions to a realistic XML framework. Further the Oxygen environment color codes elements and attributes within entries conferring a visual organization that makes presentation of the code more readable.

Effort was made to accommodate the AAT records as fully as possible within the standard SKOS framework by translating data for each AAT concept into relevant existing SKOS elements. Where elements were not readily incorporated into SKOS, further analysis of these elements offered potential solutions. The full XML document containing the records created for this study is included in an appendix. 


\section{Analysis}

Most of the syndetic references of AAT were readily incorporated into the basic elements of the SKOS framework. As SKOS was developed to address international standards of thesaurus development, this is unsurprising. The system readily accommodates simple hierarchies, preferred terminologies, and simple notations. However, the complexities of faceted classification and polyhierarchy present in AAT required development of an AAT-specific application profile in order

<skos:Concept rdf:about="www.getty.org/aat/skos/\#300006888">

<skos:prefLabel lang="en">

fortifications

<skos:sourceNote rdf:resource="www.getty.org/aat/reference/\#2000047206"/>

$<$ skos:sourceNote rdf:resource="www.getty.org/aat/reference/\#2000039651"/>

<skos:sourceNote rdf:resource="www.getty.org/aat/reference/\#2000024811"/>

<skos:sourceNote rdf:resource="www.getty.org/aat/reference/\#2000019771"/>

<skos:sourceNote rdf:resource="www.getty.org/aat/reference/\#2000031141"/>

<skos:sourceNote rdf:resource="www.getty.org/aat/reference/\#2000046684"/>

$<$ skos:sourceNote rdf:resource="www.getty.org/aat/reference/\#2000035111"/>

$<$ skos:sourceNote rdf:resource="www.getty.org/aat/reference/\#2000035251"/>

$<$ skos:prefLabel $>$

$<$ skos:altLabel lang="en">

fortification

<skos:sourceNote rdf:resource="www.getty.org/aat/reference/\#2000046657"/>

$<$ skos:sourceNote rdf:resource="www.getty.org/aat/reference/\#2000024811"/>

$</$ skos:altLabel $>$

$<$ skos:prefLabel lang="sp">fortificación</skos:prefLabel $>$

<skos:inScheme rdf:resource="http://www.getty.org/aat"/>

$<$ skos:scopeNote>

General term for any works made to oppose a small number of troops against a greater.

$</$ skos:scopeNote $>$

<skos:broadmatch rdf:resource="www.getty.org/aat/facets/\#single_built_works_by_function" />

$<$ skos:broadmatch

rdf:resource="www.getty.org/aat/facets/\#single_built_works_by_specific_type"/>

<skos:broader pref="Y" rdf:resource="www.getty.org/aat/skos/\#single_built_works" />

$<$ skos:broader pref="N" rdf:resource="www.getty.org/aat/skos/\#fortification elements"/>

$<$ skos:narrower rdf:resource="www.getty.org/aat/skos/\#antiaircraft_towers"/>

<skos:narrower rdf:resource="www.getty.org/aat/skos/\#barricades"/>

$<$ skos:narrower rdf:resource="www.getty.org/aat/skos/\#bawns"/>

$<$ skos:narrower rdf:resource="www.getty.org/aat/skos/\#castles"/>

<skos:narrower rdf:resource="www.getty.org/aat/skos/\#city_walls"/>

<skos:narrower rdf:resource="www.getty.org/aat/skos/\#coastal_fortifications"/>

$<$ skos:narrower rdf:resource="www.getty.org/aat/skos/\#field_fortifications"/>

$<$ skos:narrower rdf:resource="www.getty.org/aat/skos/\#fortresses"/>

< skos:narrower rdf:resource="www.getty.org/aat/skos/\#forts"/>

<skos:narrower rdf:resource="www.getty.org/aat/skos/\#limites"/>

$<$ skos:narrower rdf:resource="www.getty.org/aat/skos/\#plant_fortifications"/>

<skos:narrower rdf:resource="www.getty.org/aat/skos/\#redoubts"/>

$<$ skos:narrower rdf:resource="www.getty.org/aat/skos/\#stockades"/>

$<$ skos:narrower rdf:resource="www.getty.org/aat/skos/\#tighremts"/>

$</$ skos:Concept $>$

Figure 3 A sample SKOS record including exemplification of all record elements. 
to effectively capture these relationships. While simpler source and history notes are readily accomplished in SKOS, the complex notations needed for the association of sources for preferred versus alternative terminologies require some further development. Figure 3 serves as an example, incorporating all the SKOS elements described in this section.

The standard URI reference method for RDF utilizes the $r d f$ : about element to identify the unique resource. To maintain association with the standard unique identifiers in AAT, the id number was utilized as the URI for the SKOS record. Thus, the entry for the 'Castles' concept record included the rdf : about command :

<skos:Concept rdf:about="www.getty.org/aat/skos/\#300006891">, where \#300006891 is the AAT identification number. Each AAT concept term record was entered as a SKOS concept within a concept scheme referenced by a URI within the skos:inScheme element: <skos: inscheme rdf:resource="http://www.getty.org/aat"/>. Finally, descriptive elements for the syndetic references and additional notes were then nested with in the skos: concept element.

Alternative and preferred terminology were readily captured using the standard skos:altLabel and skos:prefLabel elements. These elements were present in some respect for every concept entry and most often represented spelling variants, singular vs. plural variation, and order inversion (Dark Ages vs. Ages, Dark) on the preferred label. None of the standard references required special treatment within the AAT application profile.

As AAT has recently made efforts to create a Spanish version of the thesaurus ("Tesauro de Arte \& Arquitectura," 2010), this provided an opportunity to implement a multilingual SKOS schema that could serve as a bridge for searching documents in a variety of languages. SKOS provides a standard method for identifying the language of a term via specifying an XML attribute lang. A single record can thus contain multiple translations of preferred and alternative terminologies uniting language differences within a single record:

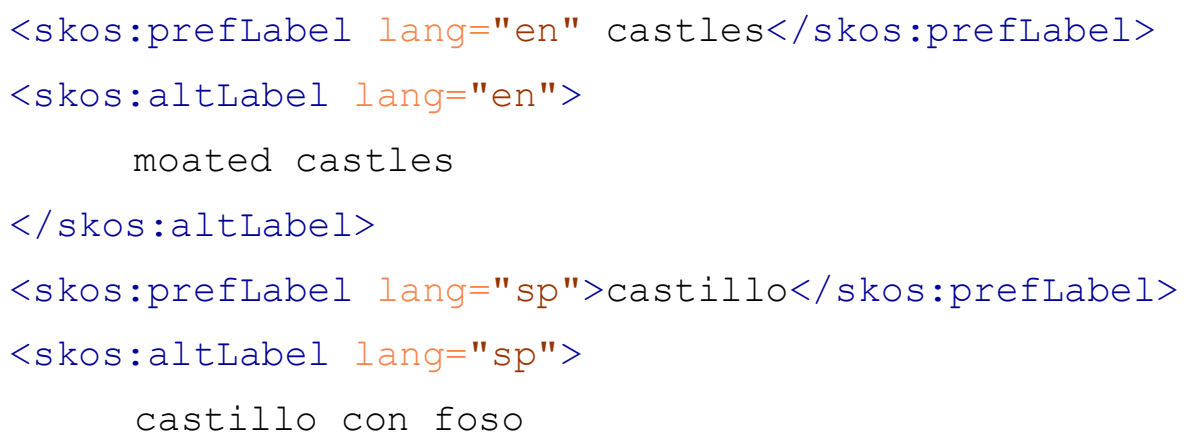




\section{$</$ skos:altLabel $>$}

Spanish entries were made for terms where available within each SKOS record.

Qualifiers of terms unexpectedly presented a challenge to the simple SKOS framework. As described above, these qualifiers are used to distinguish homonyms or to differentiate specific contexts of treatment. Other KOSs incorporate the qualifier into the label itself; however AAT explicitly dictates that qualifiers remain textually separate from the label. It was initially proposed that this exploratory AAT application profile utilize the standard XML attribute function to assign a qualifier to a label, as in the following element:

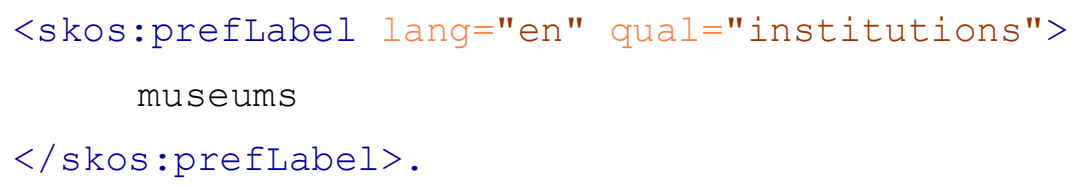

Problems arise with this method, however, since AAT allows for multiple qualifiers to be present for a single label. XML does not allow multiple specifications of the same attribute, so another method was proposed.

By opening the prefLabel element, another XML element can be inserted to capture multiple qualifiers as sister nodes to the text label:

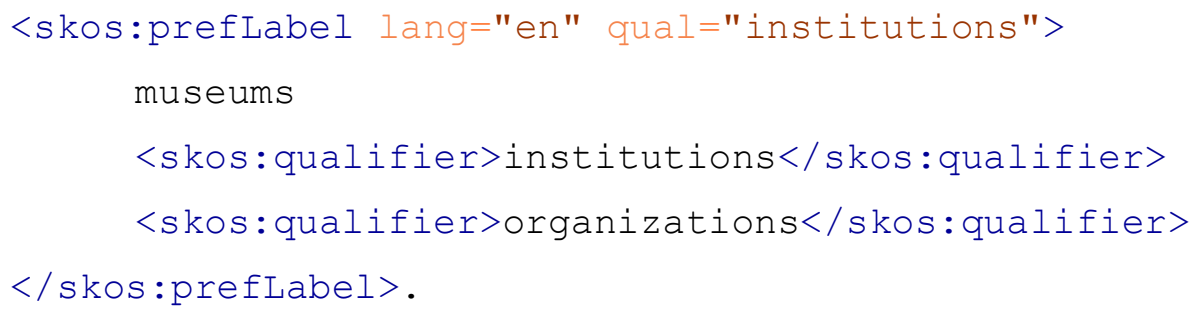

While this is far from an elegant solution codewise, it does separate the qualifier from the text, as required by AAT specifications, while retaining its association as an element of the prefLabel.

Another challenge for implementing AAT in SKOS was presented by scope notes and definitions. SKOS provides standard methods of incorporating both types of notes in a skos : concept record, but the AAT does not seem to distinguish between the two. SKOS recommends using skos: definition for the provision of a "complete explanation of the intended meaning of a concept", while skos:scopeNote is intended for "possibly partial, information about the intended meaning of a concept, especially as an indication of how the use of a concept is limited in indexing practice" 
(Isaac \& Summers, 2009, sec. 2.4). Standard treatment would require that the AAT note be inserted in one or the other of these two SKOS elements, but there were clearly situations of both sorts presented. For example, the note "can be used for substantial periods of any cultures from which little reliable data are available" listed for the concept 'Dark Ages' was clearly a treatment of scope, while the note for 'cathedrals':

The buildings housing churches in a Christian system that have an episcopal form of government, where the church is the primary church in a diocese or the seat of a diocesan bishop, archbishop, primate, patriarch, or pope. They are generally grandiose structures and house the "cathedra" or throne of the bishop (Art and Architecture Thesaurus Online).

was more clearly of the definitional type. Thus, it was left to the discretion of the record creator which of these best captured the content of the note in AAT, and the note was treated accordingly.

By far the greatest challenge for the SKOS implementation of AAT was the accurate and effective representation of the polyhierarchical elements of the thesaurus. While these could be simply represented using the skos : broader and skos : narrower elements, this simplistic coding might present problems for the computational semantic processing of these hierarchies. The more-straightforward transitive relations that hold in other ontologies might fall into violation and create conflicts in logical manipulation of the data contained within the record structure.

SKOS has been criticized elsewhere for the lack of a formal ontological framework that rigidly accommodates such computational processes (Sanchez-Alonso \& Garcia-Barriocanal, 2006), and the polyhierarchical structure of AAT would seem to exacerbate this already problematic framework.

A partial solution to this can be found in AAT's own implementation of a preferred parent for concept terms that map to multiple hierarchies. Thus, while 'ksour' exists within hierarchies for both 'villages' and 'fortifications', AAT lists 'villages' as the preferred parent. By indicating a hierarchical preference within the SKOS record, coding could avert ambiguities that might contribute to conflict when processing a node as a child of multiple parents.

It was decided that the simplest way to represent this within the SKOS record was to create a pref attribute within the SKOS application profile. Values of ' $Y$ ' or ' $N$ ' would indicate whether a declared broader reference were the preferred parent or not. For example, the 'ksour' record includes the following broader references: 


\section{<skos:broader preferred="N"rdf:resource="www.getty.org/aat/skos/\#fortresses"/> \\ $<$ skos:broader preferred="Y" rdf:resource="www.getty.org/aat/skos/\#villages"/>,}

clearly indicating 'villages' as the preferred parent concept term.

Facets also posed a challenge to the SKOS encoding of the AAT. Since facets often bridge across simpler hierarchies, a single concept term might have membership in multiple facets, or in a single facet with several different attributes, such as 'European Styles and Movements' and 'German Styles and Movements'.

It has been proposed that facets might be well-represented within SKOS as separate concept schemes, and that the skos: broadMatch element be used to link a concept in one scheme to the concept within the broader facet scheme (Suominen, Viljanen, \& Hyv \Änen). Following this proposal, a separate facet concept scheme was envisioned to contain the various AAT facets. This concept scheme, referred to by the URI rdf:resource="www.getty.org/aat/skos/facets/\# would be referenced only for broader and narrower facets, while simple conceptual hierarchical relations would be referenced via the original AAT schema URI.

Facets and subfacets would be organized within a concept scheme hierarchy of their own, and referenced from the AAT concept terms via the skos:broadMatch or skos: narrowMatch elements as suggested by Suominen et al. (n.d.).

Source notes presented another major problem for the simplistic SKOS standards. Shortcomings in the capabilities for representing documentation notes have been discussed in the literature and proposals have been made for a variety of documentations (Tennis \& Sutton, 2008), but these recommendations do not address the nature of the textual source documentation provided within AAT. Very detailed sources are provided in each record, documenting references for both preferred concept labels and alternate labels. Each of these sources is represented as a reference record within the AAT itself. Thus a single source listed for a preferred term would link to record with its own unique identifier or source ID, with brief and expanded reference citations for the source itself ("Art and Architecture Thesaurus Online," 2010).

Since the references are provided with unique identifiers, this offers an excellent starting point for framing the reference itself within RDF by referencing the listings as URIs, though not necessarily within the SKOS. URIs could be used to represent textual sources within a separate RDF schema for references. These references could be incorporated as attribute properties on a newly proposed skos: sourceNote element referring to the URI as an rdf: resource attribute: 


\section{<skos: sourcenote \\ rdf:resource="www.getty.org/aat/reference/\#2000047206"/>}

This element would be nested within the skos:prefLabel or skos:altLabel elements to capture the fact that the reference was a property of the label. While a fuller RDF representation might be more desirable for full computational purposes, this would require the establishment of URIs for each individual prefLabel and altLabel, potentially multiplying the size of the document representation ten- to one-hundred-fold. The nesting solution accomplishes a similar function, allowing accessibility for display and query within the XML environment, but does not allow the full semantic web capability offered by a more complete RDF solution. However, the establishment of URIs for reference titles, a simple process given the previous existence of Reference ID numbers, is a move toward a greater linked data environment.

This preliminary analysis of the required structure for more fully representing AAT within the SKOS framework has identified a number of elements that require further development of SKOS for fuller implementation. While the suggestions offered here are do not employ the full resources of the underlying OWL structure of SKOS discussed above, they do demonstrate the adaptability of SKOS and the ease with which schema specifics can be accommodated.

\section{Conclusion}

SKOS provides a rich framework for capturing thesauri and other KOS in semantic web framework. The RDF structure of the references not only provide for machine operability on these records, but also offer the opportunity to incorporate the URIs into the growing environment of linked data. It is important to explore implementations of SKOS in a variety of frameworks to identify weaknesses and opportunities for expansion of the framework to accommodate a range of applications.

The Art and Architecture Thesaurus is a comprehensive KOS for visual arts and architecture classification and can be utilized in a number of broader descriptive metadata schema, including widely used standards like MODS and DC. This examination of a preliminary application profile of AAT in SKOS has identified areas for further development of SKOS and has offered modest proposals for accommodating these weaknesses. Two additional SKOS concept schemes were proposed to accommodate the AAT source references and the AAT faceted classification divisions. Other minor adaptations utilized simple XML attribute and element functions to account for specificities within the AAT. While these proposals are more strongly oriented toward the XML framework within which SKOS and RDF are developed, further examination of OWL and other ontology-based languages might provide 
more powerful solutions that continue to broaden the potential for machine-processing and broadly interoperable linking of data.

As it stands, the application profile captures many of the most critical aspects of the thesaurus within the basic SKOS framework as described in the SKOS Reference (Miles \& Bechhofer, 2009) including the high-level syndetic relationships among concept terms and hierarchical structure within a relatively simple application profile. Further investigation of these characteristics could provide additional capabilities for interoperability in the web environment.

Many studies have demonstrated the capabilities of SKOS for providing an extremely interoperable framework for searching across repositories (Binding, May, \& Tudhope, 2008; Tudhope \& Binding, 2006; Tudhope, Binding, May, \& Heritage, 2008; Van Assem, Malaisé, A. Miles, \& Schreiber, 2006). By incorporating SKOS representations of AAT and other knowledge organization systems into metadata schemas, information professionals can vastly improve searchability within the linked data environment of the web.

Further opportunities in this regard would include incorporation of other KOS frameworks within the SKOS record. The inclusion of equivalence mappings for schemas like the controlled vocabulary of the Library of Congress Subject Headings or the classifications of the Dewey Decimal System, could vastly improve the retrieval of resources that are described by disparate organizations employing a variety of standards. By bridging the gaps across these silos of information organization, SKOS representation can help to realize the benefits of a more fully linked web of information. 


\section{References}

Antoniou, G., \& Harmelen, F. V. (2008). A Semantic Web Primer, 2nd Edition (2nd ed.). Cambridge, Mass.: MIT Press.

Art and Architecture Thesaurus Online. (2010). The Getty. http://www.getty.edu/research/conducting_research/vocabularies/aat/about.html

Authorities \& Vocabularies (Library of Congress). (n.d). Library of Congress. http://id.loc.gov/authorities/

Berners-Lee, T., Hendler, J., \& Lassila, O. (2001). The Semantic Web. Scientific American, 284(5), 3443. https://doi.org/10.1038/scientificamerican0501-34

Binding, C., May, K., \& Tudhope, D. (2008). Semantic interoperability in archaeological datasets: data mapping and extraction via the CIDOC CRM. In Proceedings (ECDL 2008) 12th European Conference on Research and Advanced Technology for Digital Libraries. Berlin: Springer.

Bizer, C., Cyganiak, R., \& Heath, T. (2008). How to publish linked data on the web. Presented at the 7th International Semantic Web Conference, Karlsruhe, Germany. https://doi.org/10.1145/1367497.1367760

Bizer, C., Heath, T., Ayers, D., \& Raimond, Y. (n.d.). Interlinking open data on the web. Presented at the 4th European Semantic Web Conference. http://www.eswc2007.org/pdf/demopdf/LinkingOpenData.pdf

Bradley, F. (2009, April 15). Discovering linked data. Library Journal, 48-50.

Bueno de la Fuente, G. (2008). The Simple Knowledge Organization System (SKOS) : a situation report to the HIVE Project. Chapel Hill, NC: Metadata Research Center of the School of Library and Information Systems at the University of North Carolina.

Cantara, L. (2006). Encoding controlled vocabularies for the Semantic Web using SKOS Core. OCLC Systems \& Services, 22(2), 111 - 114. https://doi.org/10.1108/10650750610663996

Coyle, K. (2008). Meaning, technology, and the Semantic Web. The Journal of Academic Librarianship, 34(3), 263-264. https://doi.org/10.1016/j.acalib.2008.03.010

Coyle, K. (2009, April 15). Making connections. Library Journal, 44-47.

Harper, C. A., \& Tillett, B. B. (2007). Library of Congress controlled vocabularies and their application to the Semantic Web. Cataloging \& Classification Quarterly, 43(3), 47-68. https://doi.org/10.1300/J104v43n03_03

Hodge, G. (2000). Systems of knowledge organization for digital libraries: beyond traditional authority files. Washington, D.C.: Digital Library Federation, Council on Library and Information Resources. 
International Organization for Standardization. (1985). Guidelines for the establishment and development of multilingual thesauri (Text No. ISO 5964). http://www.iso.org/iso/catalogue detail.htm?csnumber=12159

International Organization for Standardization. (1986). Guidelines for the establishment and development of monolingual thesauri (Text No. ISO 2788). http://www.iso.org/iso/catalogue detail.htm?csnumber=7776

Isaac, A., \& Summers, E. (2009). SKOS Simple Knowledge Organization System primer. W3C. Retrieved March 1, 2010, from http://www.w3.org/TR/skos-primer/

Jupp, S., Stevens, R., Bechhofer, S., Yesilada, Y., \& Kostkova, P. (2008). Knowledge representation for web navigation. Semantic Web Applications and Tools for the Life Sciences. http://ftp.informatik.rwth-aachen.de/Publications/CEUR-WS/Vol-435/paper03.pdf

Kim, H. L., Passant, A., Breslin, J. G., Scerri, S., \& Decker, S. (2008). Review and alignment of tag ontologies for semantically-linked data in collaborative tagging spaces. In 2008 IEEE International Conference on Semantic Computing (pp. 315-322). https://doi.org/10.1109/ICSC.2008.79

Lacasta, J., Nogueras-Iso, J., Lopez-Pellicer, F. J., Muro-Medrano, P. R., \& Zarazaga-Soria, F. J. (2007). ThManager: An open source tool for creating and visualizing SKOS. Information Technology and Libraries, 26(3), 39-50. https://doi.org/10.6017/ital.v26i3.3274

Limpens, E. F., Gandon, F., \& Buffa, M. (2009). Linking Folksonomies and Ontologies for Supporting Knowledge Sharing: a State of the Art. Projet ISICIL : Intégration Sémantique de l'Information par des Communautés d'Intelligence en Ligne.

Macgregor, G., \& McCulloch, E. (2006). Collaborative tagging as a knowledge organisation and resource discovery tool. Library Review, 55(5), 291-300. https://doi.org/10.1108/00242530610667558

McBride, B. (2004a). RDF primer. W3C. http://www.w3.org/TR/2004/REC-rdf-primer-20040210/

McBride, B. (2004b). RDF vocabulary description language 1.0: RDF Schema. W3C. http://www.w3.org/TR/rdf-schema/

McGuinness, D., \& van Harmelen, F. (2004). OWL Web Ontology Language Overview. W3C. http://www.w3.org/TR/owl-features/

Mikhalenko, P. (2005). Introducing SKOS. https://www.xml.com/pub/a/2005/06/22/skos.html

Miles, A., Matthews, B., Beckett, D., Brickley, D., Wilson, M., \& Rogers, N. (2005). SKOS: A language to describe simple knowledge structures for the web. In XTech 2005 Conference Proceedings. 
Miles, A., Matthews, B., Wilson, M., \& Brickley, D. (2005). SKOS Core: Simple knowledge organisation for the web. In Proceedings of the International Conference on Dublin Core and Metadata Applications (pp. 12-15).

Miles, A., \& Bechhofer, S. (2009). SKOS Simple Knowledge Organization System Reference. W3C. http://www.w3.org/TR/skos-reference/

Miles, A., \& Brickley, D. (2004). SKOS Mapping vocabulary specification. W3C. http://www.w3.org/2004/02/skos/mapping/spec/2004-11-11.html

Panzer, M., \& Zeng, M. L. (2009). Modeling Classification Systems in SKOS: Some Challenges and BestPractice Recommendations. Presented at the International Conference on Dublin Core and Metadata Applications, Seoul, South Korea. http://www.nla.gov.au/lis/stndrds/grps/acoc/documents/EPC132-38.1.doc

Robinson, E. A. (ms.). SKOS and the Semantic Web: knowledge organization for metadata interoperability. https://soar.usa.edu/other/11/

Sanchez-Alonso, S., \& Garcia-Barriocanal, E. (2006). Making use of upper ontologies to foster interoperability between SKOS concept schemes. Online Information Review, 30(3), 263-277. https://doi.org/10.1108/14684520610675799

Sauermann, L., \& Cyganiak, R. (2008). Cool URIs for the Semantic Web. W3C. http://www.w3.org/TR/cooluris/\#hashuri

Suominen, O., Viljanen, K., \& Hyv $\backslash$ Änen, (ms.) E.User-centric faceted search for semantic portals. The Semantic Web: Research and Applications, 356-370. https://doi.org/10.1007/978-3-540-72667-8_26

Tennis, J. T. (2005). SKOS and the ontogenesis of vocabularies. In Proceedings of the International Conference on Dublin Core and Metadata Applications, Madrid, Spain (Vol. 2005, pp. 12-15). http://purl.oclc.org/metadataresearch/search.jsp

Tennis, J. T., \& Sutton, S. A. (2008). Extending the simple knowledge organization system for concept management in vocabulary development applications. Journal of the American Society for Information Science and Technology, 59(1), 25-37. https://doi.org/10.1002/asi.20702

Tesauro de Arte \& Arquitectura. (2010). http://www.aatespanol.cl/taa/tesauro/default.asp? a=224

Tudhope, D., \& Binding, C. (2008). Machine Understandable Knowledge Organization Systems (No. Project no.507618). DELOS: Network Excellence on Digital Libraries.

Tudhope, D., Binding, C., May, K., \& Heritage, E. (2008). Semantic interoperability issues from a case study in archaeology. In Semantic Interoperability in the European Digital Library, Proceedings of the First International Workshop SIEDL (pp. 88-99). 
Van Assem, M., Malaisé, V., Miles, A., \& Schreiber, G. (2006). A method to convert thesauri to SKOS. Lecture Notes in Computer Science, 4011, 95. https://doi.org/10.1007/11762256 10

Zeng, M. L., \& Chan, L. M. (2004). Trends and issues in establishing interoperability among knowledge organization systems. Journal of the American Society for Information Science, 55(5), 377-395. https://doi.org/10.1002/asi.10387 


\section{Appendix: XML/RDF Document of SKOS AAT Records}

$<$ ?xml version=" 1.0 " encoding="UTF-8"? $>$

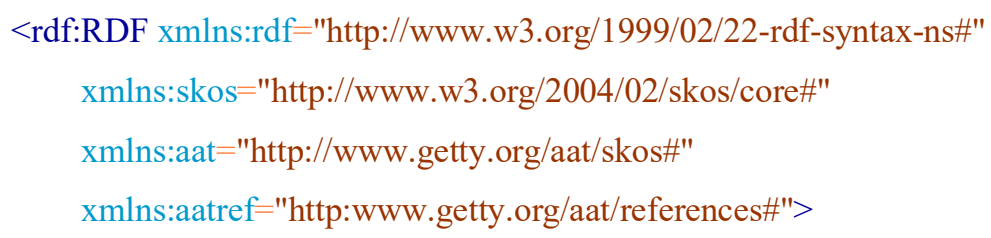


<skos:altLabel lang="en"> qasrs $<$ /skos:altLabel $>$

$<$ skos:altLabel lang="en">moated castles $</$ skos:altLabel $>$

<skos:altLabel lang="en"> qasrs $<$ /skos:altLabel $>$

<skos:prefLabel lang="sp">castillo</skos:prefLabel>

<skos:altLabel lang="sp"> qasr</skos:altLabel>

<skos:altLabel lang="sp">castillo con foso</skos:altLabel $>$

<skos:altLabel lang="sp">châtelet</skos:altLabel>

<skos:inScheme rdf:resource="http://www.getty.org/aat"/>

<skos:definition>

Buildings or groups of buildings intended primarily to serve as a fortified residence of a prince or nobleman.

$</$ skos:definition $>$

$<$ skos:broader rdf:resource="www.getty.org/aat/skos/\#fortifications"/>

$</$ skos:Concept $>$

<skos:Concept rdf:about="www.getty.org/aat/skos/\#300006888">

<skos:prefLabel lang="en">

fortifications

<skos:sourceNote rdf:resource="www.getty.org/aat/reference/\#2000047206"/>

<skos:sourceNote rdf:resource="www.getty.org/aat/reference/\#2000039651"/>

<skos:sourceNote rdf:resource="www.getty.org/aat/reference/\#2000024811"/>

<skos:sourceNote rdf:resource="www.getty.org/aat/reference/\#2000019771"/>

<skos:sourceNote rdf:resource="www.getty.org/aat/reference/\#2000031141"/>

<skos:sourceNote rdf:resource="www.getty.org/aat/reference/\#2000046684"/>

<skos:sourceNote rdf:resource="www.getty.org/aat/reference/\#2000035111"/>

<skos:sourceNote rdf:resource="www.getty.org/aat/reference/\#2000035251"/>

$</$ skos:prefLabel $>$

<skos:altLabel lang="en">

fortification

<skos:sourceNote rdf:resource="www.getty.org/aat/reference/\#2000046657"/>

<skos:sourceNote rdf:resource="www.getty.org/aat/reference/\#2000024811"/>

$</$ skos:altLabel $>$

$<$ skos:prefLabel lang="sp">fortificación</skos:prefLabel $>$

<skos:inScheme rdf:resource="http://www.getty.org/aat"/>

<skos:scopeNote>

General term for any works made to oppose a small number of troops against a greater.

$</$ skos:scopeNote $>$ 


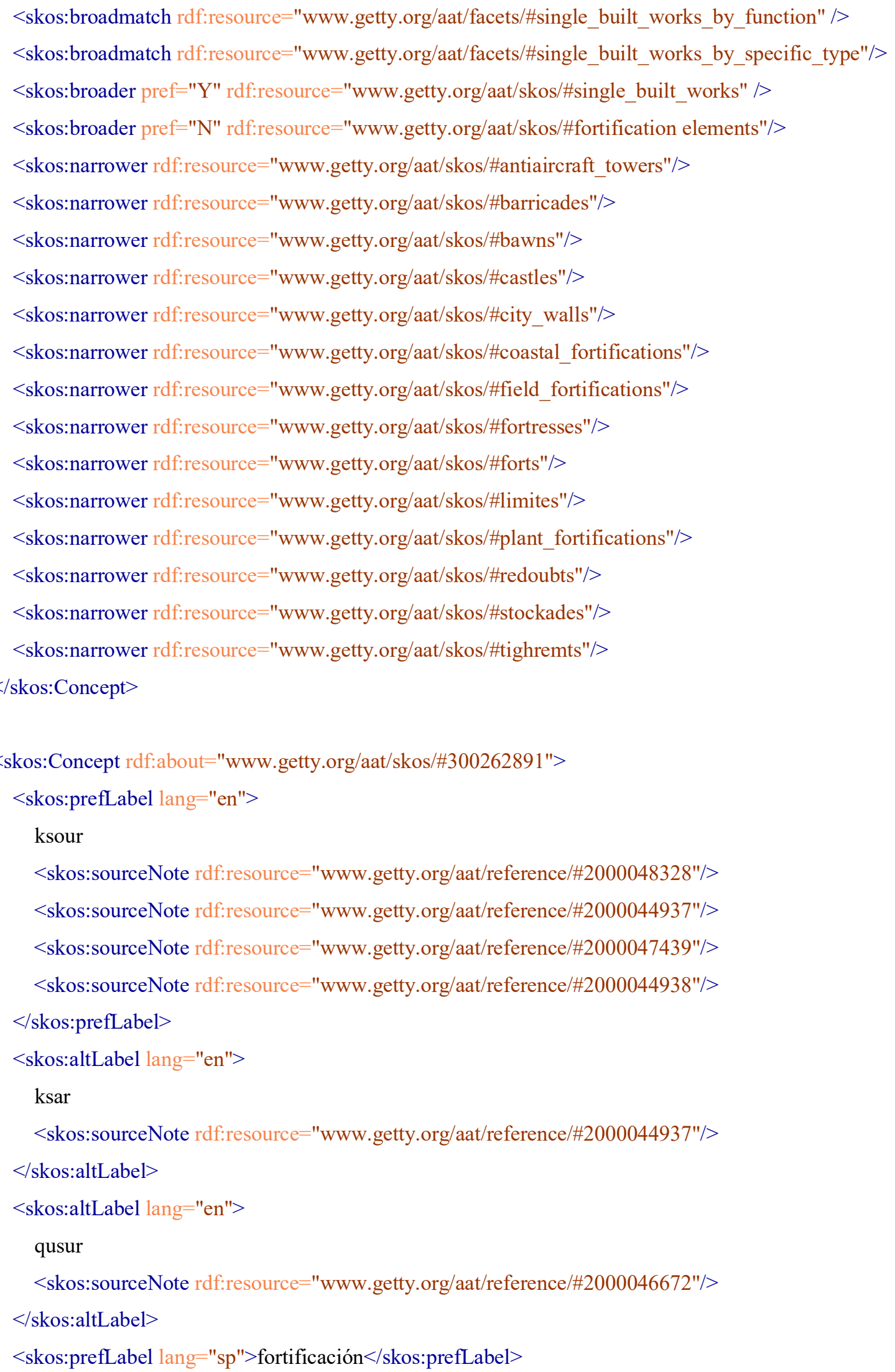


<skos:inScheme rdf:resource="http://www.getty.org/aat"/>

$<$ skos:definition>

Fortified farming villages found primarily among the Berber peoples in Morocco, Algeria, and Tunisia; mostly constructed at oases, along northeastern and southern edges of the Atlas mountains.

$</$ skos:definition $>$

$<$ skos:broader preferred="N" rdf:resource="www.getty.org/aat/skos/\#fortresses"/>

$<$ skos:broader preferred="Y" rdf:resource="www.getty.org/aat/skos/\#villages"/>

$<$ skos:Concept $>$

<skos:Concept rdf:about="www.getty.org/aat/skos/\#300002485">

$<$ skos:prefLabel lang="en" >

defensive walls

<skos:sourceNote rdf:resource="www.getty.org/aat/reference/\#2000010761"/>

$</$ skos:prefLabel $>$

$<$ skos:altLabel lang="en">

defensive wall

<skos:sourceNote rdf:resource="www.getty.org/aat/reference/\#2000046717"/>

$</$ skos:altLabel $>$

$<$ skos:altLabel lang="en">

walls, defensive

<skos:sourceNote rdf:resource="www.getty.org/aat/reference/\#2000046717"/>

$<$ skos:altLabel $>$

$<$ skos:prefLabel lang="sp">muralla defensiva $<$ skos:prefLabel $>$

<skos:inScheme rdf:resource="http://www.getty.org/aat"/>

$<$ skos:scopeNote $>$ Use broadly for all sorts of walls constructed to resist attack. $<$ skos:scopeNote $>$

$<$ skos:broader pref="Y" rdf:resource="www.getty.org/aat/skos/\#architectural_elements" />

$<$ skos:broadMatch

rdf:resource="www.getty.org/aat/skos/facets/\#defensive_walls_and_defensive_wall_components"/>

<skos:narrower rdf:resource="www.getty.org/aat/skos/\#breastworks"/>

$<$ skos:narrower rdf:resource="www.getty.org/aat/skos/\#curtain_walls"/>

$<$ skos:narrower rdf:resource="www.getty.org/aat/skos/\#enceintes"/>

$<$ skos:narrower rdf:resource="www.getty.org/aat/skos/\#honmaru"/>

<skos:narrower rdf:resource="www.getty.org/aat/skos/\#murus_gallicus"/>

$<$ skos:narrower rdf:resource="www.getty.org/aat/skos/\#ramparts"/>

$<$ skos:narrower rdf:resource="www.getty.org/aat/skos/\#valla"/>

$<$ /skos:Concept $>$ 


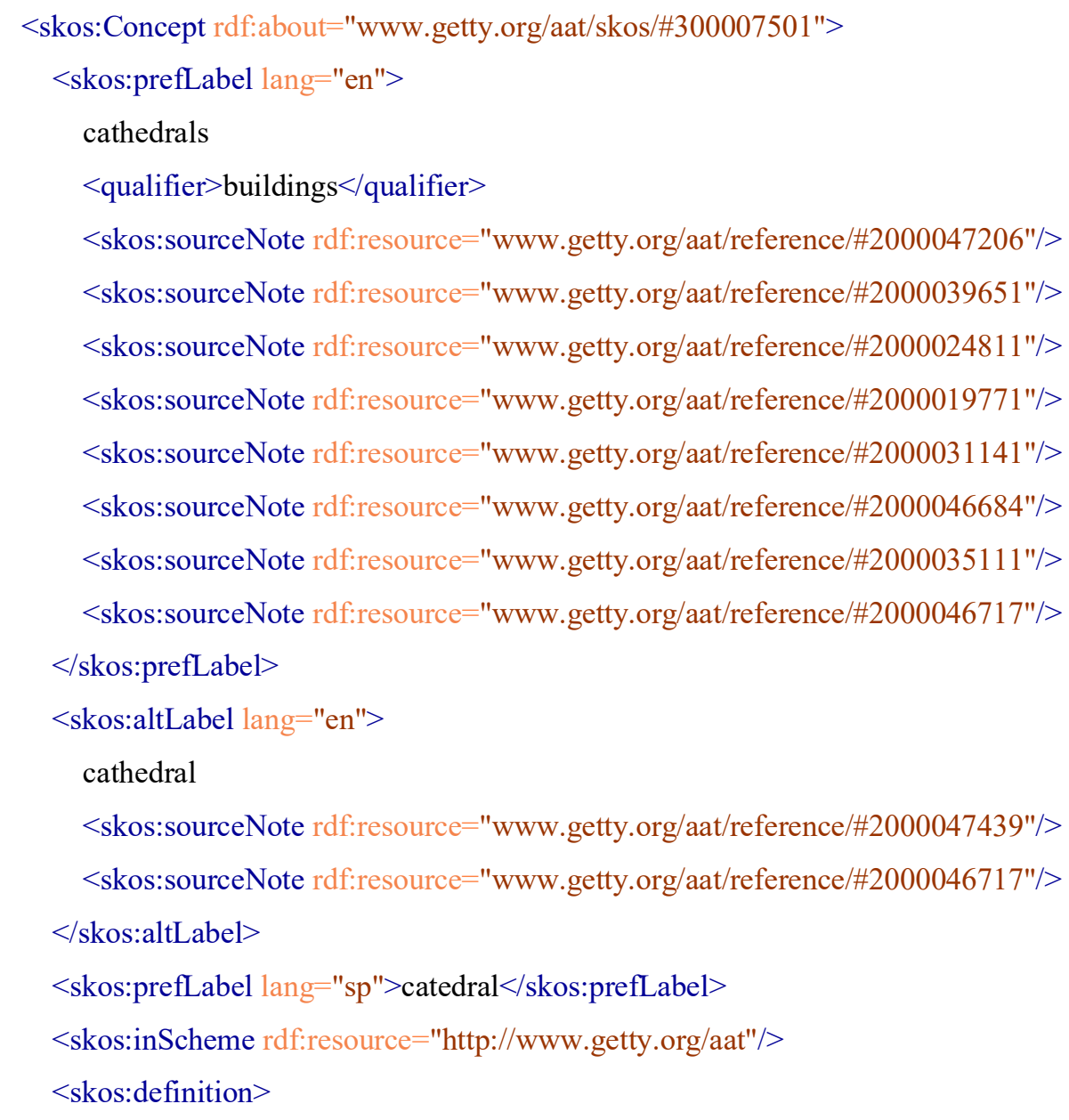

The buildings housing churches in a Christian system that have an episcopal form of government, where the church is the primary church in a diocese or the seat of a diocesan bishop, archbishop, primate, patriarch, or pope. They are generally grandiose structures and house the "cathedra" or throne of the bishop.

$</$ skos:definition $>$

<skos:broadMatch

rdf:resource="www.getty.org/aat/skos/facets/\#church_buildings_by_location_or_context"/>

<skos:broader pref="Y" rdf:resource="www.getty.org/aat/skos/\#churches" />

$</$ skos:Concept $>$

<skos:Concept rdf:about="www.getty.org/aat/skos/\#300004809">

<skos:prefLabel lang="en">

skyscrapers

$<$ aat:qualifier $>$ buildings $</$ aat:qualifier $>$

<skos:sourceNote rdf:resource="www.getty.org/aat/reference/\#2000046717"/>

<skos:sourceNote rdf:resource="www.getty.org/aat/reference/\#2000010761"/>

<skos:sourceNote rdf:resource="www.getty.org/aat/reference/\#2000024811"/> 


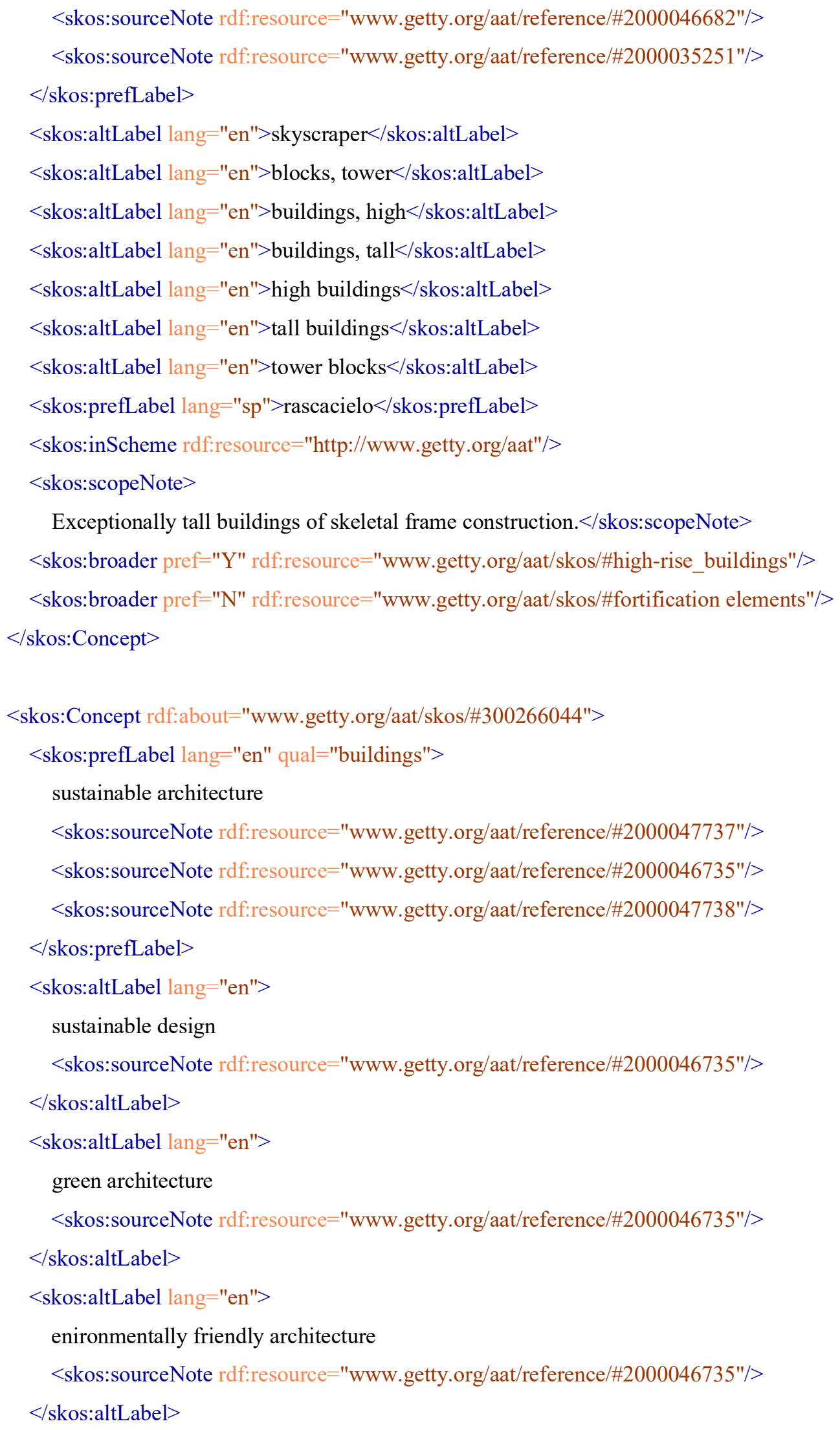

Exceptionally tall buildings of skeletal frame construction. $</$ skos:scopeNote $>$

<skos:broader pref="Y" rdf:resource="www.getty.org/aat/skos/\#high-rise_buildings"/> 
<skos:prefLabel lang="sp">arquitectura sustentable</skos:prefLabel>

<skos:inScheme rdf:resource="http://www.getty.org/aat"/>

$<$ skos:definition $>$

Structure design that is specifically environmentally conscious, taking into account construction methods and materials that are locally available as well as the building's efficient use of resources, including systems of heating, cooling, power, water, and waste. Provides affordable, adequate shelter with minimal negative effect on the local and global environment; may be replicated and locally maintained.

$</$ skos:definition $>$

<skos:broadMatch rdf:resource="www.getty.org/aat/skos/facets/\#environmental_concepts"/>

<skos:broader pref="Y" rdf:resource="www.getty.org/aat/skos/\#architecture genres"/>

<skos:related rdf:resource="www.getty.org/aat/skos/\#green design"/>

$</$ skos:Concept $>$

<skos:Concept rdf:about="www.getty.org/aat/skos/\#300068844">

<skos:prefLabel lang="en">

use

<skos:sourceNote rdf:resource="www.getty.org/aat/reference/\#2000046693"/>

<skos:sourceNote rdf:resource="www.getty.org/aat/reference/\#2000048366"/>

<skos:sourceNote rdf:resource="www.getty.org/aat/reference/\#2000046684"/>

<skos:sourceNote rdf:resource="www.getty.org/aat/reference/\#2000042061"/>

$<$ skos:prefLabel>

<skos:altLabel lang="en">

utilization

<skos:sourceNote rdf:resource="www.getty.org/aat/reference/\#2000042061"/>

$</$ skos:altLabel $>$

<skos:prefLabel lang="sp">uso</skos:prefLabel>

<skos:altLabel lang="sp"/>

<skos:inScheme rdf:resource="http:/www.getty.org/aat"/>

$<$ skos:definition $>$ The ability or permission to, or the method or manner of employing, occupying, applying or exploiting the value of something. $</$ skos:definition $>$

<skos:broadMatch rdf:resource="www.getty.org/aat/skos/facets/\#functional_concepts"/>

<skos:narrower rdf:resource="www.getty.org/aat/skos/\#adaptive_reuse"/>

<skos:narrower rdf:resource="www.getty.org/aat/skos/\#mixed_use"/>

<skos:narrower rdf:resource="www.getty.org/aat/skos/\#multiuse"/>

$</$ skos:Concept $>$

<skos:Concept rdf:about="www.getty.org/aat/skos/\#300056392"> 


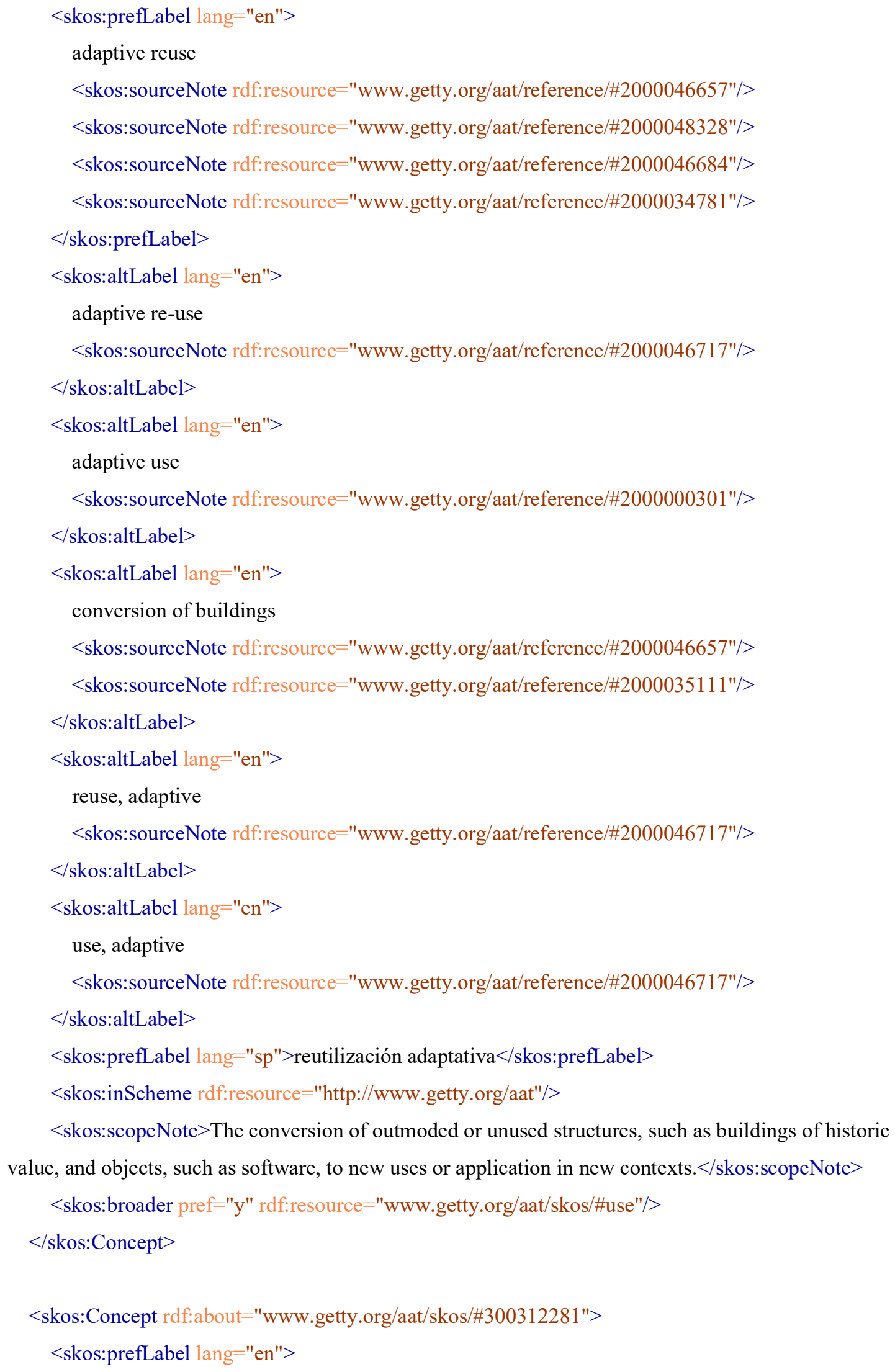




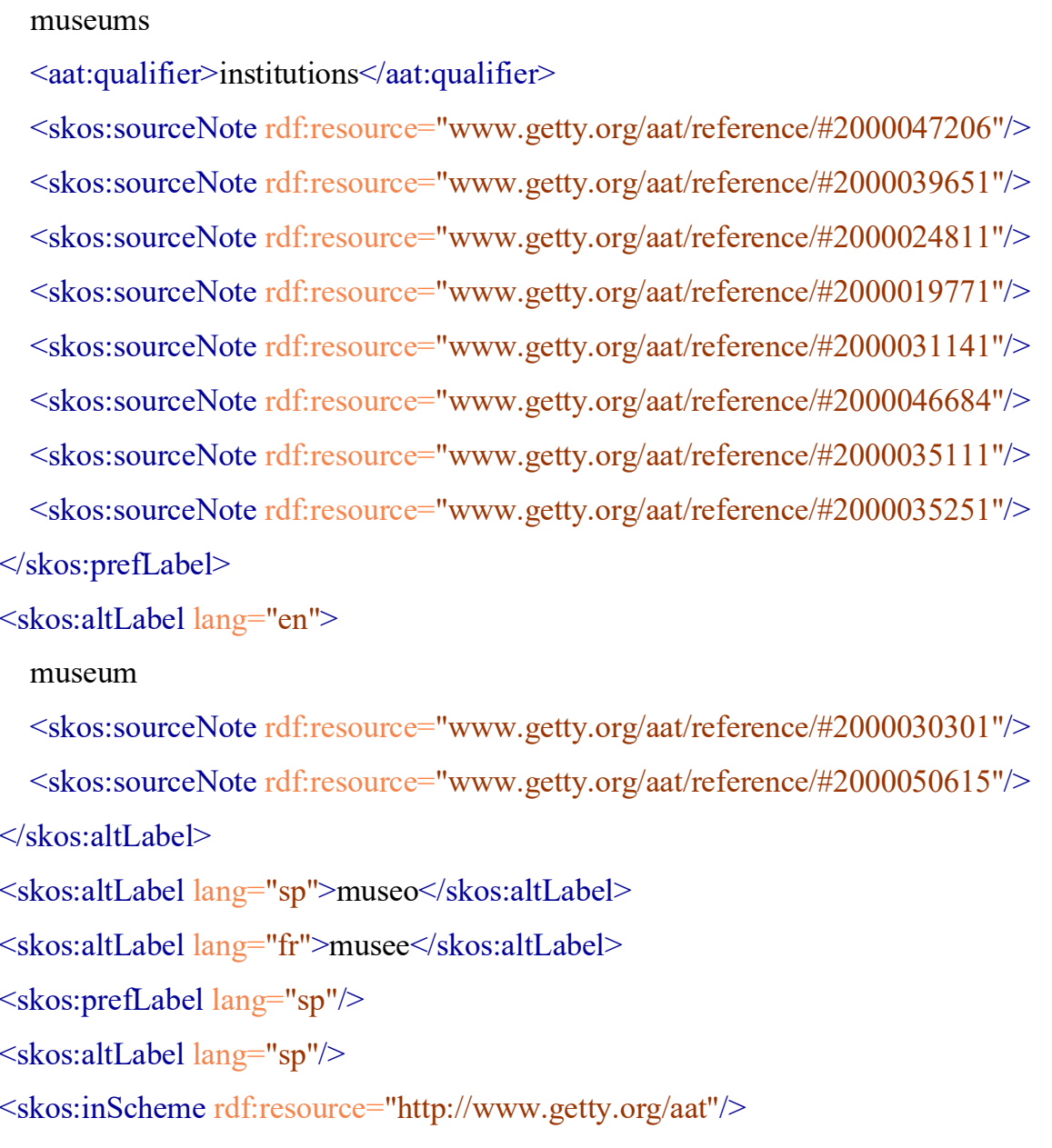

$<$ skos:scopeNote>Institutions that maintain places where objects of value such as works of art, antiquities, scientific specimens, or other artifacts are housed and displayed for public benefit. An institution devoted to the procurement, care, documentation, study and display of objects of lasting interest or value. $</$ skos:scopeNote $>$

$<$ skos:broadMatch rdf:resource="www.getty.org/aat/skos/facets/\#institutions_by activity"/>

<skos:related rdf:resource="www.getty.org/aat/skos/\#museology"/>

$<$ skos:related rdf:resource="www.getty.org/aat/skos/\#collections"/>

$<$ skos:Concept $>$

<skos:Concept rdf:about="www.getty.org/aat/skos/\#300005768">

$<$ skos:prefLabel lang="en">

museums

$<$ aat:qualifier $>$ buildings $<$ /aat:qualifier $>$

$<$ skos:sourceNote rdf:resource="www.getty.org/aat/reference/\#2000047206"/>

<skos:sourceNote rdf:resource="www.getty.org/aat/reference/\#2000039651"/>

<skos:sourceNote rdf:resource="www.getty.org/aat/reference/\#2000024811"/> 
<skos:sourceNote rdf:resource="www.getty.org/aat/reference/\#2000019771"/>

<skos:sourceNote rdf:resource="www.getty.org/aat/reference/\#2000031141"/>

<skos:sourceNote rdf:resource="www.getty.org/aat/reference/\#2000046684"/>

<skos:sourceNote rdf:resource="www.getty.org/aat/reference/\#2000035111"/>

<skos:sourceNote rdf:resource="www.getty.org/aat/reference/\#2000035251"/>

$<$ skos:prefLabel>

<skos:altLabel lang="en">

museum

<skos:sourceNote rdf:resource="www.getty.org/aat/reference/\#2000030301"/>

<skos:sourceNote rdf:resource="www.getty.org/aat/reference/\#2000050615"/>

$</$ skos:altLabel $>$

$<$ skos:related $>$ museum architecture $<$ /skos:related $>$

$<$ skos:altLabel lang="sp">museo $</$ skos:altLabel $>$

<skos:inScheme rdf:resource="http://www.getty.org/aat"/>

$<$ skos:scopeNote $>$

Refers to buildings, groups of buildings, or spaces within buildings where objects of value such as works of art, antiquities, scientific specimens, or other artifacts are housed and displayed for public benefit.

$</$ skos:scopeNote $>$

<skos:broader pref="y" rdf:resource="www.getty.org/aat/skos/\#exhibition_buildings"/>

<skos:related rdf:resource="www.getty.org/aat/skos/\#museology"/>

<skos:related rdf:resource="www.getty.org/aat/skos/\#collections"/>

<skos:related rdf:resource="www.getty.org/aat/skos/\#museums"/>

<skos:related rdf:resource="www.getty.org/aat/skos/\#collections"/>

$</$ skos:Concept $>$

<skos:Concept rdf:about="www.getty.org/aat/skos/\#300001280">

<skos:prefLabel lang="en">

domes

$<$ qualifier $>$ architectural element $<$ /qualifier $>$

<skos:sourceNote rdf:resource="www.getty.org/aat/reference/\#2000046657"/>

<skos:sourceNote rdf:resource="www.getty.org/aat/reference/\#2000024811"/>

<skos:sourceNote rdf:resource="www.getty.org/aat/reference/\#2000024811"/>

<skos:sourceNote rdf:resource="www.getty.org/aat/reference/\#2000032401"/>

<skos:sourceNote rdf:resource="www.getty.org/aat/reference/\#2000033931"/>

<skos:sourceNote rdf:resource="www.getty.org/aat/reference/\#2000046682"/>

<skos:sourceNote rdf:resource="www.getty.org/aat/reference/\#2000035111"/>

<skos:sourceNote rdf:resource="www.getty.org/aat/reference/\#2000035251"/> 


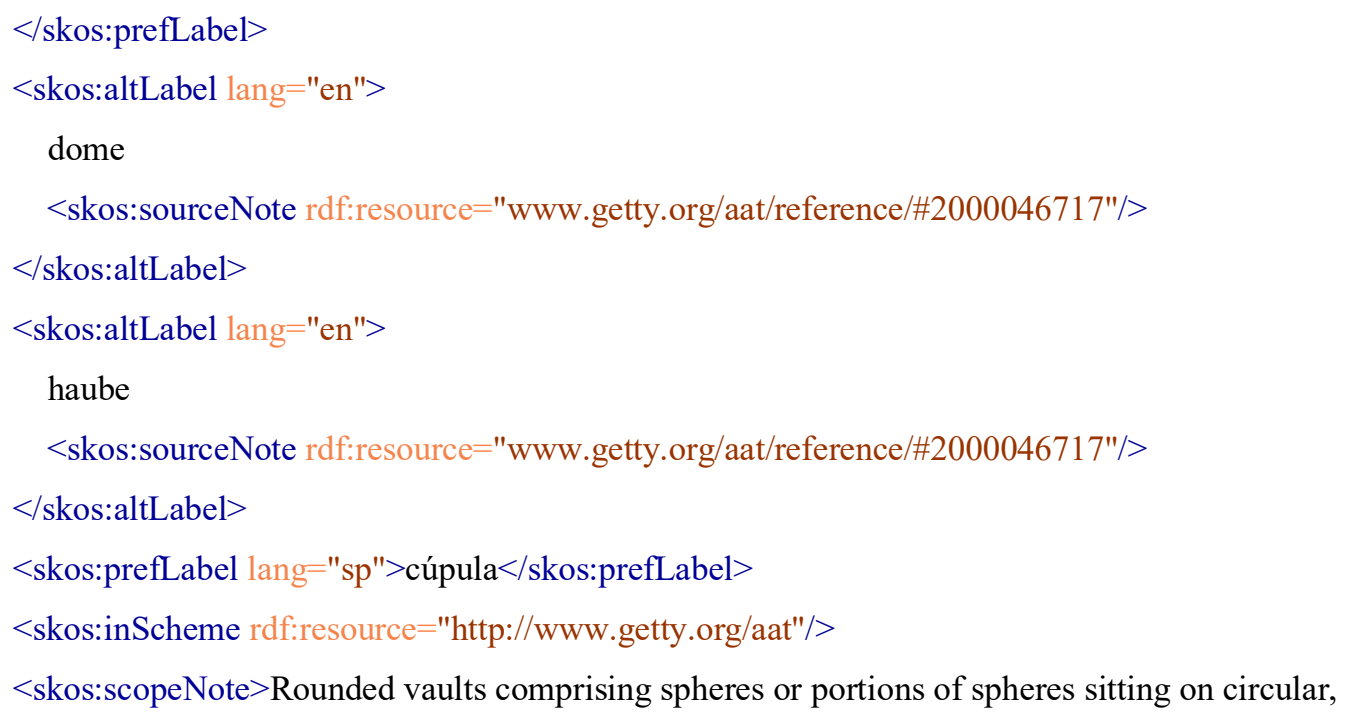
elliptical, or polygonal bases, forming the roof of any building or part of a building. They are constructed so that they exert equal thrust in all directions. $</$ skos:scopeNote $>$

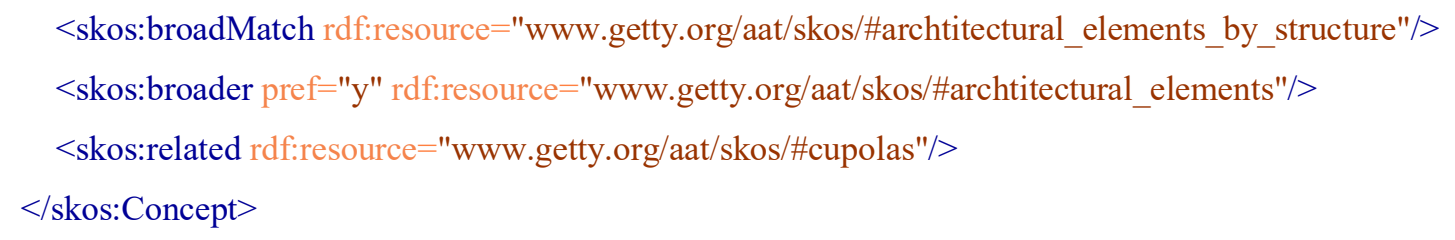


imitated for structural or decorative use on other objects. $</$ skos:scopeNote $>$

$<$ skos:broadMatch pref="y"

rdf:resource="www.getty.org/aat/skos/\#components_by_specific_context"/>

<skos:narrower rdf:resource="www.getty.org/aat/skos/\#structural_elements"/>

$<$ skos:narrower rdf:resource="www.getty.org/aat/skos/\#surface_elements"/>

$<$ skos:narrower rdf:resource="www.getty.org/aat/skos/\#openings"/>

$<$ skos:narrower rdf:resource="www.getty.org/aat/skos/\#barriers"/>

<skos:narrower rdf:resource="www.getty.org/aat/skos/\#culminating_and_edge_ornaments"/>

$<$ skos:narrower rdf:resource="www.getty.org/aat/skos/\#circulation_elements"/>

<skos:narrower rdf:resource="www.getty.org/aat/skos/\#heating_and_cooking_elements"/>

<skos:narrower rdf:resource="www.getty.org/aat/skos/architectural_elements_by_building_type>\#"/> $<$ skos:Concept $>$

<skos:Concept rdf:about="www.getty.org/aat/skos/\#300002660">

$<$ skos:prefLabel lang="en">

pavilions

$<$ qualifier $>$ building divisions $<$ /qualifier $>$

<skos:sourceNote rdf:resource="www.getty.org/aat/reference/\#2000046657"/>

$<$ skos:sourceNote rdf:resource="www.getty.org/aat/reference/\#2000047206"/>

$<$ skos:sourceNote rdf:resource="www.getty.org/aat/reference/\#2000046717"/>

<skos:sourceNote rdf:resource="www.getty.org/aat/reference/\#2000016821"/>

$<$ skos:prefLabel $>$

$<$ skos:altLabel lang="en">

pavilion

<skos:sourceNote rdf:resource="www.getty.org/aat/reference/\#2000046717"/>]

$<$ skos:altLabel $>$

$<$ skos:altLabel lang="en">

pavillions

<skos:sourceNote rdf:resource="www.getty.org/aat/reference/\#2000046682"/>

$<$ skos:sourceNote rdf:resource="www.getty.org/aat/reference/\#2000042061"/>

$<$ /skos:altLabel $>$

$<$ skos:altLabel lang="en">

avant-corps

<skos:sourceNote rdf:resource="www.getty.org/aat/reference/\#2000047206"/>

$<$ skos:sourceNote rdf:resource="www.getty.org/aat/reference/\#2000005871"/>

$<$ skos:sourceNote rdf:resource="www.getty.org/aat/reference/\#2000018341"/>

<skos:sourceNote rdf:resource="www.getty.org/aat/reference/\#2000035871"/> 
$</$ skos:altLabel $>$

$<$ skos:altLabel lang="en" $>$ facade, pavilions $<$ skos:altLabel $>$

$<$ skos:altLabel lang="en">pavilions, façade $<$ skos:altLabel $>$

$<$ skos:prefLabel lang="sp">pabellón</skos:prefLabel>

$<$ skos:inScheme rdf:resource="http://www.getty.org/aat"/>

$<$ skos:scopeNote>Refers to both for the parts of buildings that project outward from the rest, especially common as corner, central, or terminal features in French architecture, and for the detached or semidetached units into which a building (as a hospital) is sometimes divided. $</$ skos:scopeNote $>$

$<$ skos:broadMatch rdf:resource="www.getty.org/aat/skos/facets/\#components_by_specific_context"/> $<$ skos:Concept $>$

<skos:Concept rdf:about="www.getty.org/aat/skos/\#300008090">

$<$ skos:prefLabel lang="en">gardens

<skos:sourceNote rdf:resource="www.getty.org/aat/reference/\#2000047206"/>

$<$ skos:sourceNote rdf:resource="www.getty.org/aat/reference/\#2000039651"/>

$<$ skos:sourceNote rdf:resource="www.getty.org/aat/reference/\#2000024811"/>

<skos:sourceNote rdf:resource="www.getty.org/aat/reference/\#2000019771"/>

$<$ skos:sourceNote rdf:resource="www.getty.org/aat/reference/\#2000031141"/>

<skos:sourceNote rdf:resource="www.getty.org/aat/reference/\#2000046684"/>

$<$ skos:sourceNote rdf:resource="www.getty.org/aat/reference/\#2000035111"/>

<skos:sourceNote rdf:resource="www.getty.org/aat/reference/\#2000035251"/>

$<$ skos:prefLabel $>$

$<$ skos:altLabel lang="en">

garden

<skos:sourceNote rdf:resource="www.getty.org/aat/reference/\#2000030301"/>

$<$ skos:sourceNote rdf:resource="www.getty.org/aat/reference/\#2000050615"/></skos:altLabel $>$

$<$ skos:prefLabel lang="sp">jardín</skos:prefLabel>

$<$ skos:inScheme rdf:resource="http://www.getty.org/aat"/>

$<$ skos:scopeNote $>$ none $</$ skos:scopeNote $>$

$<$ skos:broader pref="Y" rdf:resource="www.getty.org/aat/skos/\#open_spaces"/>

$<$ skos:broadMatch rdf:resource="www.getty.org/aat/skos/facets/\#open_spaces_by form"/>

<skos:narrowMatch rdf:resource="www.getty.org/aat/skos/facets/\#gardens_by_form"/>

<skos:narrowMatch rdf:resource="www.getty.org/aat/skos//facets/\#gardens_by_location"/>

<skos:narrowMatch rdf:resource="www.getty.org/aat/skos/facets/\#gardens_by_product"/>

$<$ skos:narrowMatch rdf:resource="www.getty.org/aat/skos/facets/\#gardens_by_ownership"/>

$<$ skos:related rdf:resource="www.getty.org/aat/skos/\#horticulture"/>

$<$ skos:Concept $>$ 


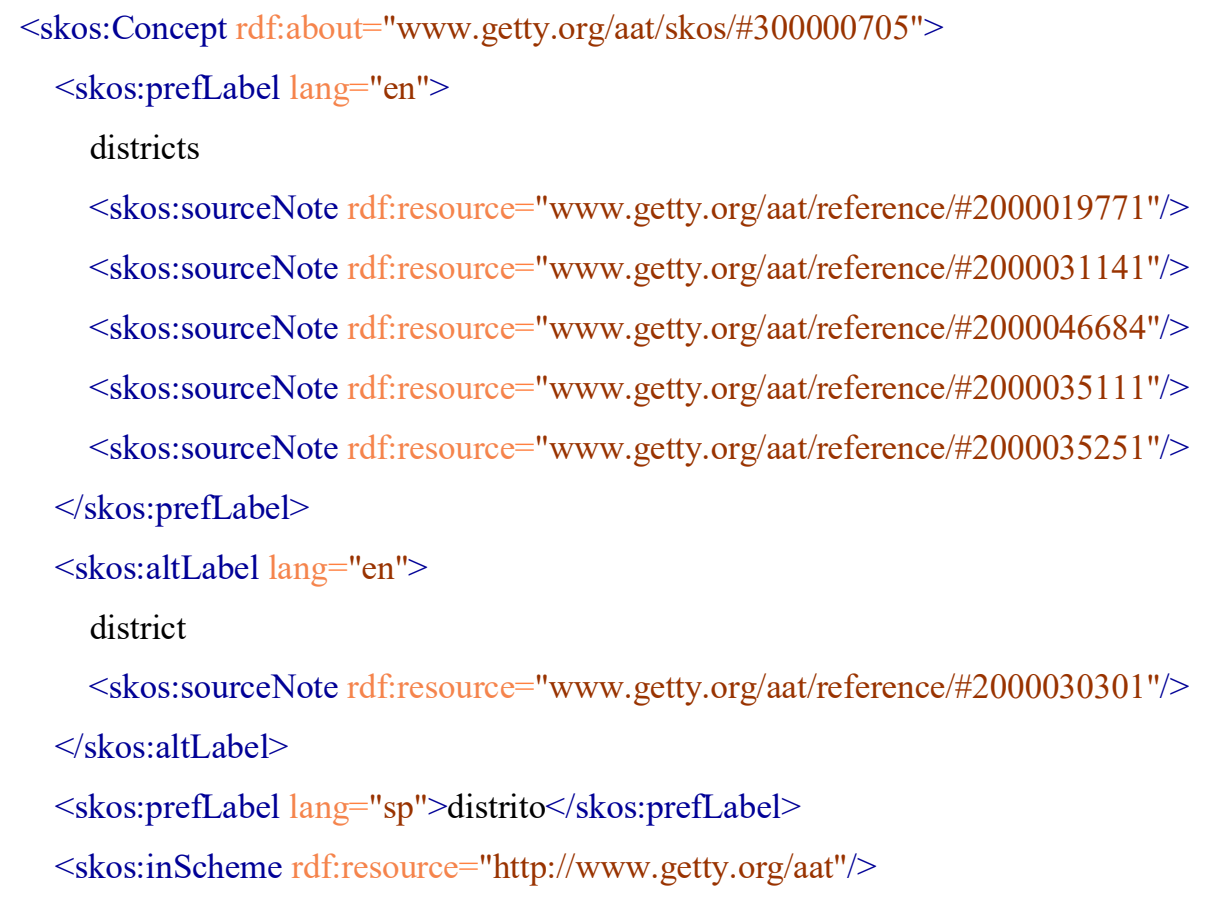

$<$ skos:scopeNote $>$ Territorial divisions as for administrative or electoral purposes, or settlement areas with distinguishing characteristics. $</$ skos:scopeNote $>$

<skos:broader pref="Y" rdf:resource="www.getty.org/aat/skos/\#built_complexes_and_districts"/> <skos:narrowMatch rdf:resource="www.getty.org/aat/skos/facets/\#districts_by_function"/> <skos:narrowMatch rdf:resource="www.getty.org/aat/skos/facets/\#districts_by_location"/> <skos:narrowMatch rdf:resource="www.getty.org/aat/skos/facets/\#districts_by_condition"/> $</$ skos:Concept $>$

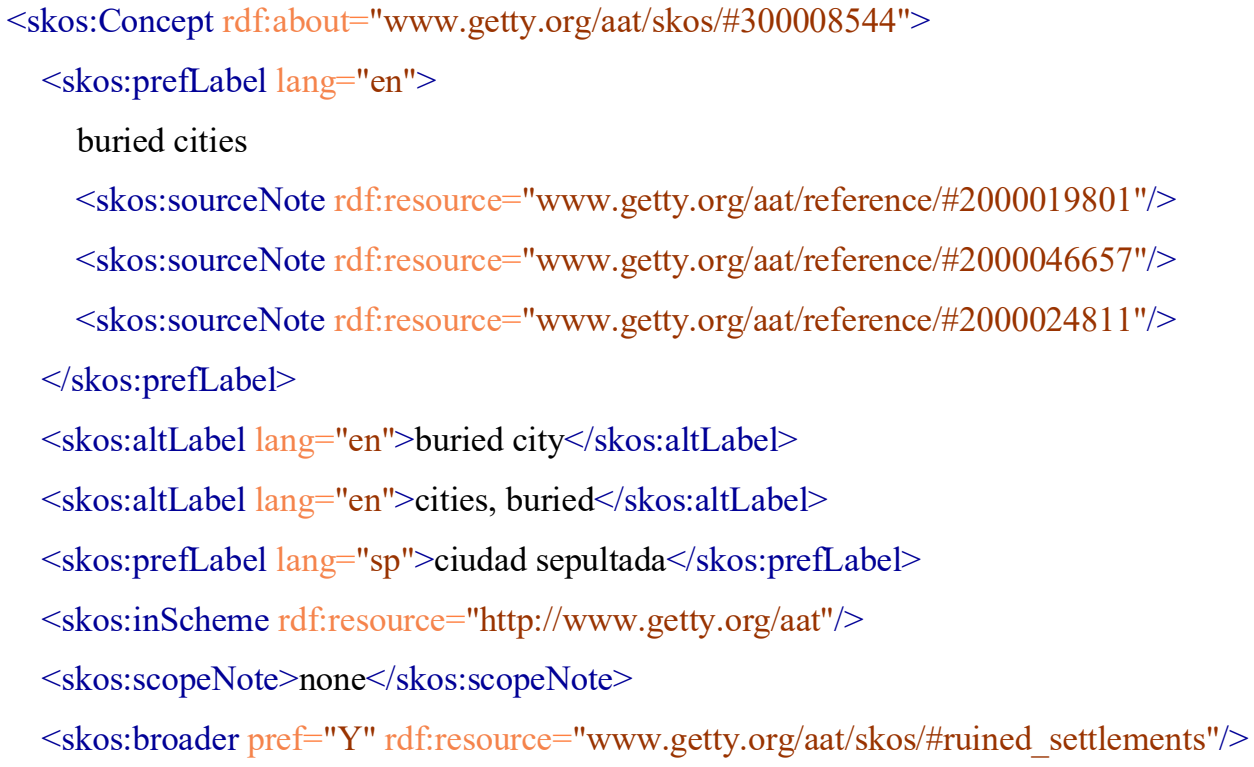




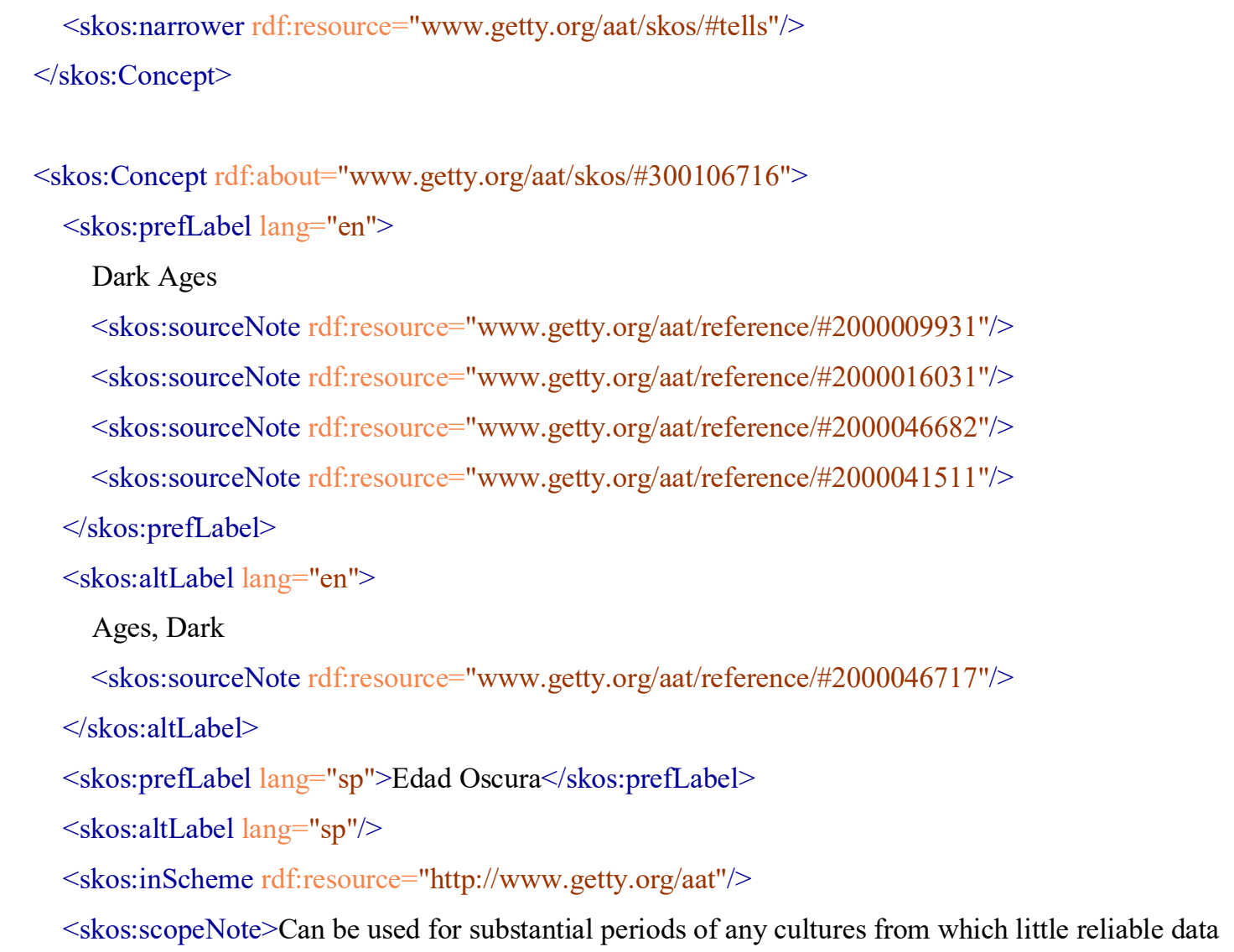
are available. $</$ skos:scopeNote $>$

<skos:broadMatch pref="Y"

rdf:resource="www.getty.org/aat/skos/facets/\#styles_and_periods_by_general_era"/>

<skos:broadMatch pref="Y"

rdf:resource="www.getty.org/aat/skos/facets/\#styles_and_periods_by_era"/>

$</$ skos:Concept $>$

<skos:Concept rdf:about="www.getty.org/aat/skos/\#300312281">

<skos:prefLabel lang="en">

bearing walls

<skos:sourceNote rdf:resource="www.getty.org/aat/reference/\#2000032311"/>

<skos:sourceNote rdf:resource="www.getty.org/aat/reference/\#2000010761"/>

<skos:sourceNote rdf:resource="www.getty.org/aat/reference/\#2000046682"/>

<skos:sourceNote rdf:resource="www.getty.org/aat/reference/\#2000014901"/>

<skos:sourceNote rdf:resource="www.getty.org/aat/reference/\#2000000051"/>

<skos:sourceNote rdf:resource="www.getty.org/aat/reference/\#2000033931"/>

<skos:sourceNote rdf:resource="www.getty.org/aat/reference/\#2000046682"/>

<skos:sourceNote rdf:resource="www.getty.org/aat/reference/\#2000027101"/> 


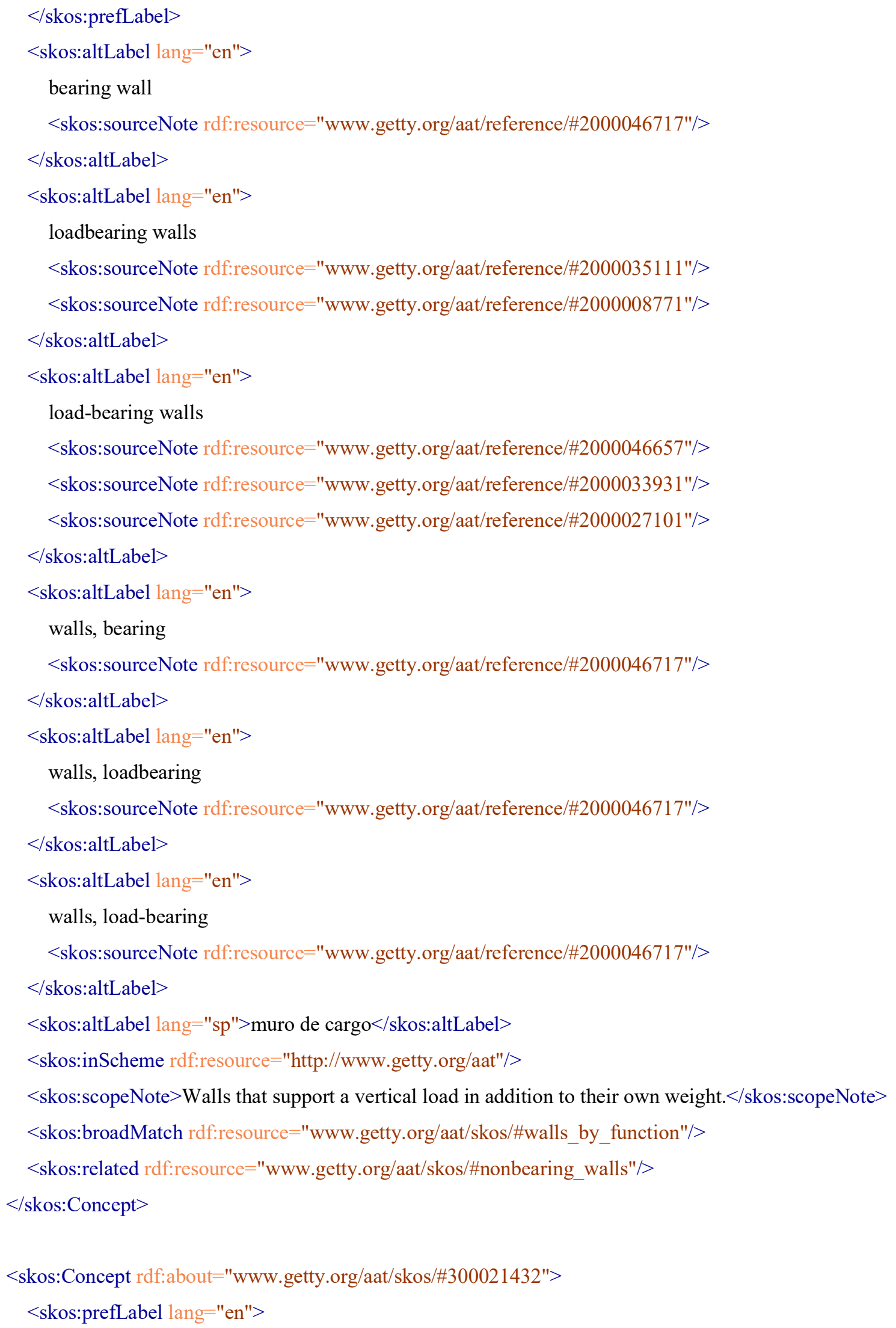




\section{Bauhaus}

<skos:sourceNote rdf:resource="www.getty.org/aat/reference/\#2000046657"/>

<skos:sourceNote rdf:resource="www.getty.org/aat/reference/\#2000011141"/>

<skos:sourceNote rdf:resource="www.getty.org/aat/reference/\#2000046682"/>

<skos:sourceNote rdf:resource="www.getty.org/aat/reference/\#2000030761"/>

<skos:sourceNote rdf:resource="www.getty.org/aat/reference/\#2000030811"/>

<skos:sourceNote rdf:resource="www.getty.org/aat/reference/\#2000035111"/>

<skos:sourceNote rdf:resource="www.getty.org/aat/reference/\#2000035251"/>

<skos:sourceNote rdf:resource="www.getty.org/aat/reference/\#2000043551"/>

$<$ /skos:prefLabel $>$

<skos:altLabel lang="sp">Bauhaus $</$ skos:altLabel $>$

<skos:inScheme rdf:resource="http://www.getty.org/aat"/>

$<$ skos:scopeNote>Refers to the German School of art, design, and architecture active in Weimar from 1919 to 1925, in Dessau from 1925 to 1932, and in Berlin from 1932 to 1933. Training students in both fine art and craftsmanship, it produced objects and building designs intended for mass production using simple, geometric forms. $</$ skos:scopeNote $>$

<skos:broader rdf:resource="www.getty.org/aat/skos/\#styles_and_periods"/>

<skos:broadMatch rdf:resource="www.getty.org/aat/skos//facets/\#styles_and_periods_by_region"/>

<skos:broadMatch

rdf:resource="www.getty.org/aat/skos//facets/\#styles_and_movements_by_region"/>

<skos:broader rdf:resource="www.getty.org/aat/skos/\#European_styles_and_periods"/>

<skos:broader rdf:resource="www.getty.org/aat/skos/\#modern German_styles_and_movements"/>

$</$ skos:Concept $>$

<skos:Concept rdf:about="www.getty.org/aat/skos/\#300241583">

<skos:prefLabel lang="en">

components

$<$ qualifier $>$ objects $<$ /qualifier $>$

<skos:sourceNote rdf:resource="www.getty.org/aat/reference/\#2000042061"/>

$</$ skos:prefLabel $>$

<skos:altLabel lang="en">

component

<skos:sourceNote rdf:resource="www.getty.org/aat/reference/\#2000042061"/>

$</$ skos:altLabel $>$

<skos:altLabel lang="en">

parts

<skos:sourceNote rdf:resource="www.getty.org/aat/reference/\#2000048328"/> 


\footnotetext{
<skos:sourceNote rdf:resource="www.getty.org/aat/reference/\#2000031141"/>

<skos:sourceNote rdf:resource="www.getty.org/aat/reference/\#2000042061"/>

$</$ skos:altLabel $>$

<skos:altLabel lang="sp">componentes $<$ /skos:altLabel $>$

<skos:inScheme rdf:resource="http://www.getty.org/aat"/>

$<$ skos:scopeNote $>$ Constituent parts of objects or structures. $</$ skos:scopeNote $>$

<skos:broader facet="objects" rdf:resource="www.getty.org/aat/skos/\#objects"/>

$</$ skos:Concept $>$

<skos:Concept rdf:about="www.getty.org/aat/skos/\#300189645">

<skos:prefLabel lang="en">

urban design

<skos:sourceNote rdf:resource="www.getty.org/aat/reference/\#2000040861"/>

<skos:sourceNote rdf:resource="www.getty.org/aat/reference/\#2000033151"/>

<skos:sourceNote rdf:resource="www.getty.org/aat/reference/\#2000039651"/>

<skos:sourceNote rdf:resource="www.getty.org/aat/reference/\#2000010621"/>

<skos:sourceNote rdf:resource="www.getty.org/aat/reference/\#2000048328"/>

<skos:sourceNote rdf:resource="www.getty.org/aat/reference/\#2000024811"/>

<skos:sourceNote rdf:resource="www.getty.org/aat/reference/\#2000001281"/>

$<$ skos:prefLabel>

<skos:altLabel lang="en">

design, urban

<skos:sourceNote rdf:resource="www.getty.org/aat/reference/\#2000046717"/>

$</$ skos:altLabel $>$

<skos:altLabel lang="sp">diseño urbano</skos:altLabel $>$

<skos:inScheme rdf:resource="http:/www.getty.org/aat"/>

$<$ skos:scopeNote>Refers to the field of study and practice that falls between and overlaps architecture and city and regional planning, and whose concern is the specific design and contextual integration of city buildings and spaces; includes expertise in, for example, landscape architecture, urban sociology, environmental psychology, and law. $</$ skos:scopeNote $>$

<skos:broader pref="Y" rdf:resource="www.getty.org/aat/skos/\#architecture"/>

<skos:broader pref="N" rdf:resource="www.getty.org/aat/skos/\#urban_studies"/>

<skos:broadMatch rdf:resource="www.getty.org/aat/skos//facets/\#planning_disciplines"/>

$</$ skos:Concept $>$

<skos:Concept rdf:about="www.getty.org/aat/skos/\#300189645">

<skos:prefLabel lang="en">
} 
urban history

<skos:sourceNote rdf:resource="www.getty.org/aat/reference/\#2000010621"/>

<skos:sourceNote rdf:resource="www.getty.org/aat/reference/\#2000045253"/>

<skos:sourceNote rdf:resource="www.getty.org/aat/reference/\#2000039651"/>

$<$ skos:sourceNote rdf:resource="www.getty.org/aat/reference/\#2000035111"/>

$<$ skos:sourceNote rdf:resource="www.getty.org/aat/reference/\#2000041071"/>

$<$ skos:sourceNote rdf:resource="www.getty.org/aat/reference/\#2000045252"/>

$<$ skos:sourceNote rdf:resource="www.getty.org/aat/reference/\#2000046692"/>

$<$ skos:prefLabel $>$

$<$ skos:altLabel lang="en">

history, urban

<skos:sourceNote rdf:resource="www.getty.org/aat/reference/\#2000046717"/>

$</$ skos:altLabel $>$

$<$ skos:altLabel lang="en">

history of cities

<skos:sourceNote rdf:resource="www.getty.org/aat/reference/\#2000035111"/>

$</$ skos:altLabel $><$ skos:altLabel lang="en">

cities, historty of

<skos:sourceNote rdf:resource="www.getty.org/aat/reference/\#2000046717"/>

$</$ skos:altLabel $>$

$<$ skos:altLabel lang="sp"> historia urbana</skos:altLabel>

$<$ skos:inScheme rdf:resource="http://www.getty.org/aat"/>

$<$ skos:defnition $>$ The history of cities and towns, the urbanization of human life, and all aspects of urban life over time. $</$ skos:defnition>

<skos:broader pref="Y" rdf:resource="www.getty.org/aat/skos/\#urban_studies"/>

$<$ skos:broader pref="N" rdf:resource="www.getty.org/aat/skos/\#history"/>

$<$ skos:broadMatch rdf:resource="www.getty.org/aat/skos//facets/\#histor_and_related_disciplines"/> $</$ skos:Concept $>$

<skos:Concept rdf:about="www.getty.org/aat/skos/\#300265318">

$<$ skos:prefLabel lang="en">

blob architecture

<skos:sourceNote rdf:resource="www.getty.org/aat/reference/\#2000047094"/>

$<$ skos:sourceNote rdf:resource="www.getty.org/aat/reference/\#2000047097"/>

$<$ skos:prefLabel>

$<$ skos:altLabel lang="en">

blobitecture 


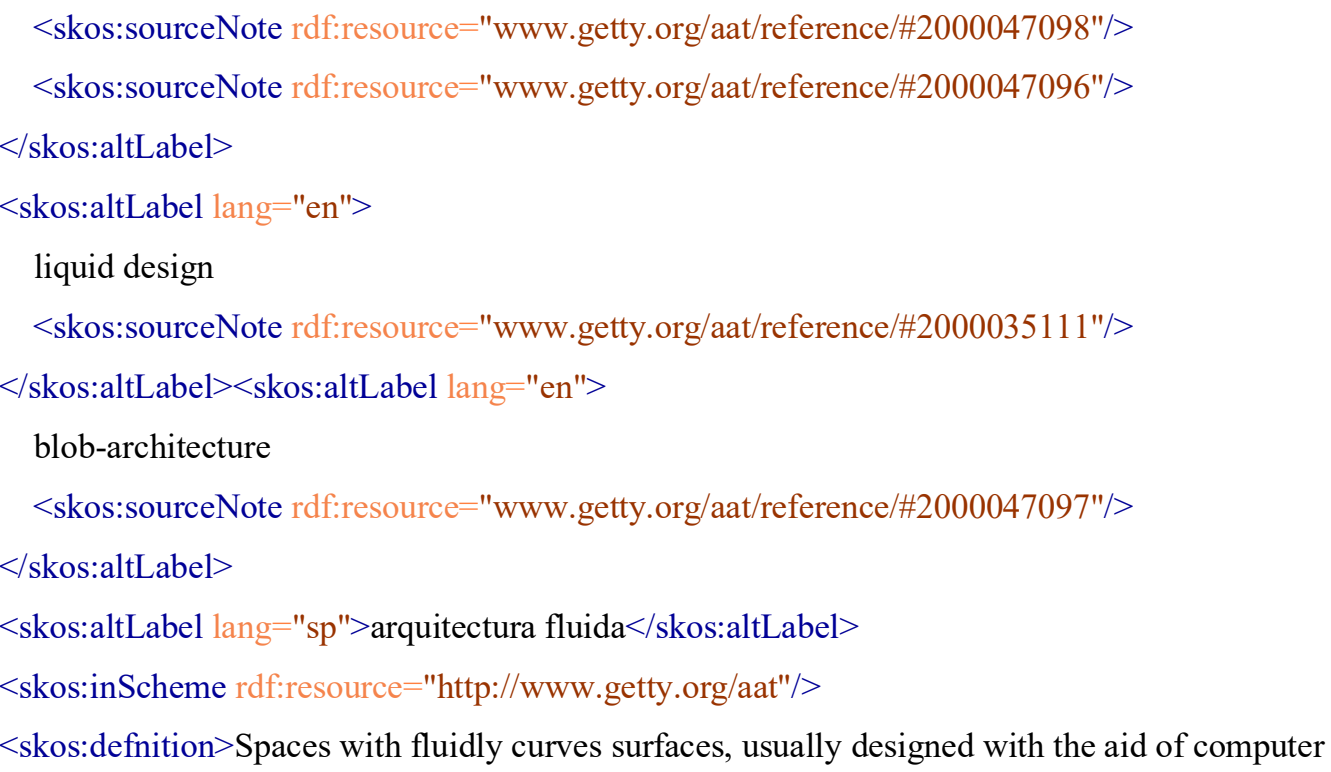
software. $</$ skos:defnition $>$

<skos:broadMatch rdf:resource="www.getty.org/aat/skos//facets/\#architecture_genres"/> $</$ skos:Concept $>$

<skos:Concept rdf:about="www.getty.org/aat/skos/\#300259487">

<skos:prefLabel lang="en">

New Urbanism

<skos:sourceNote rdf:resource="www.getty.org/aat/reference/\#2000048328"/>

<skos:sourceNote rdf:resource="www.getty.org/aat/reference/\#2000021961"/>

<skos:sourceNote rdf:resource="www.getty.org/aat/reference/\#2000033691"/>

$</$ skos:prefLabel $>$

<skos:altLabel lang="en">

Urbanism, New

<skos:sourceNote rdf:resource="www.getty.org/aat/reference/\#2000046717"/>

$</$ skos:altLabel $>$

$<$ skos:altLabel lang="sp">Nuevo Urbanismo</skos:altLabel $>$

<skos:inScheme rdf:resource="http://www.getty.org/aat"/>

<skos:defnition> The planning movement in the United States of the 1990s espousing a return to traditional small-town design features for newly constructed suburbs or redeveloped urban neighborhoods. To describe the architectural elements of the movement, incorporating such features as pedestrian spaces, mass transit, and mixed land use, use "Neotraditional."</skos:defnition>

<skos:broader rdf:resource="www.getty.org/aat/skos//facets/\#Styles and Periods"/>

<skos:broadMatch

rdf:resource="www.getty.org/aat/skos//facets/\#modern_North_American_styles_and_movements"/> 
$</$ skos:Concept $>$

$</$ rdf:RDF $>$ 